\title{
Accessible Triple-Phase Boundary Length: A Performance Metric to Account for Transport Pathways in Heterogeneous Electrochemical Materials
}

\author{
A. Nakajo ${ }^{\mathrm{a}, \mathrm{b}}$, A.P. Cocco ${ }^{\mathrm{a}}$, M.B. DeGostin ${ }^{\mathrm{a}}$, A.A. Peracchio ${ }^{\mathrm{a}}$, B.N. Cassenti ${ }^{\mathrm{a}}$, M. Cantoni ${ }^{\mathrm{c}}, \mathrm{J}$. \\ Van herle ${ }^{\mathrm{b}}$, W.K.S. Chiu ${ }^{\mathrm{a},{ }^{*}}$ \\ ${ }^{\text {a }}$ Department of Mechanical Engineering, University of Connecticut, Storrs, USA \\ ${ }^{\mathrm{b}}$ Fuelmat Group, Faculty of Engineering Sciences and Technology STI, Ecole Polytechnique \\ Fédérale de Lausanne, Lausanne, Switzerland \\ c Interdisciplinary Centre for Electron Microscopy, Ecole Polytechnique Fédérale de \\ Lausanne, Lausanne, Switzerland \\ *Corresponding author: wchiu@engr.uconn.edu
}

\begin{abstract}
The performance of materials for electrochemical energy conversion and storage depends upon the number of electrocatalytic sites available for reaction and their accessibility by the transport of reactants and products. For solid oxide fuel/electrolysis cell materials, standard 3D measurements such as connected triple-phase boundary (TPB) length and effective transport properties partially inform on how local geometry and network topology causes variability in TPB accessibility. A new measurement, the accessible TPB, is proposed to quantify these effects in detail and characterize material performance.
\end{abstract}

The approach probes the reticulated pathways to each TPB using an analytical electrochemical fin model applied to a 3-D discrete representation of the heterogeneous structure provided by skeleton-based partitioning. The method is tested on artificial and real structures imaged by 3-D x-ray and electron microscopy. The accessible TPB is not uniform and the pattern varies depending upon the structure. Connected TPBs can be even passivated. The sensitivity to manipulations of the local 3-D geometry and topology that standard measurements cannot capture is demonstrated. The clear presence of preferential pathways showcases a non-uniform utilization of the 3-D structure that potentially affects the performance and the resilience to alterations due to degradation phenomena in electrochemical energy storage and conversion devices. 


\section{Introduction}

The advances of 3-D imaging methods during the last decade have significantly improved the understanding of the relationships between the microstructure and performance of heterogeneous materials for electrochemical energy conversion and storage [1-3]. Nowadays, metric and topological properties are measured directly on 3-D reconstructions of the materials imaged by e.g. x-ray nanotomography (XNT) or focused ion beam-scanning electron microscopy (FIB-SEM) serial sectioning [2-8]. This capability provides insight into how the assembly of the constituents, i.e. the morphology and topology of the phases and their interfaces, impact the material functionality, which is essential for the rational design of heterogeneous materials [9].

The relationship between the triple-phase boundary (TPB) length in solid oxide fuel or electrolysis cell (SOFC/SOEC) composite materials and the polarization resistance has been first ascertained using patterned electrode experiments [10-12]. The performance of SOFC/SOEC materials therefore largely depends upon the TPB length formed in the structure and the ability to transport ions, electrons and gas species to these TPB reaction sites. At present, the two functions are treated as partially coupled in several SOFC/SOEC performance and degradation studies. The approach is based upon the measurement of the effective transport properties of the phases and of the connected TPB length, which is the subset that has connected pathways for ions, electrons and gas species to flow to the electrolyte, current collection and gas channel, respectively. The development of methods for the accurate measurement of these properties, among others, using 3-D reconstruction has been the subject of significant effort $[2,4,5,7,13-15]$. The measurements are then applied to materials produced by different fabrication routes or subjected to aging or specific treatments and, in some cases, their effects on the electrode polarization resistance are assessed using continuum electrode models. This approach has contributed to significant improvements of 
our understanding of the detrimental effects of microstructural coarsening, contamination or redox cycling in SOFC/SOEC materials $[6,8,16-23]$.

A main limitation of approaches based on averaged (e.g. effective) properties is that all the TPBs are treated as equally accessible. The visual inspection of 3-D imaging data suggests that this assumption is questionable and that significant local information is lost. In contrast, the TPB tortuosity and TPB critical pathway radius have been recently proposed for the characterization of the transport pathways to TPBs [24]. This approach, based on image processing, provides new insight into the factors that control the electrochemical performance of heterogeneous materials, but it is based on purely geometric concepts that highlight two specific and mostly local properties of the reticulate pathways, i.e. the shortest path length and the smallest constriction that must be passed through to access a TPB. Simulations based on lattice Boltzmann or finite element method that use as computational domain the imaged and meshed 3-D structures $[4,25]$ are capable of quantifying accurately the access to TPB, including the combined effects of local 3-D geometrical features and topology of the microstructure. However, attempts to define dedicated TPB properties that inform on material performance and durability have been limited so far [4], partly because of the high computational requirements.

An analytical electrochemical fin model (ECF) has been developed as a screening tool for material design [26-29]. The method consists in representing the heterogeneous structure as a network of segments. Each segment is characterized for simplification into 1-D axisymmetric shape profiles for which analytical solutions to the combined surface charge transport and charge transport problem exist. This simplification yields computation times that are at least three orders of magnitude faster than that of FE simulations. The computation time does practically not exceed a few seconds for typical volume samples obtained by $\mathrm{x}$-ray nanotomography (XNT) or focused ion beam - scanning electron microscopy (FIB-SEM). 
Comparisons with 3-D finite-element simulation results of artificial and real structures imaged by x-ray nanotomography and electrochemical impedance spectroscopy (EIS) measurements have shown that adequate sensitivity to local 3-D geometry and network topology is retained $[28,29]$. With such capabilities, the ECF method is not restricted to the calculations of standard transport properties or polarization resistance. The low computational requirement resulting from the implementation of analytical solutions extends its use as a method to probe heterogeneous structures, in a way similar to shortest-path algorithms to measure metrics relevant in graph theory, such as node betweeness, for instance [30]. However, the current approach is fully informed about all 3-D pathways in the complex structure.

In this study, the accessible TPB is proposed as a new measurement to characterize the access to TPBs in materials with multiple material phases and 3-D heterogeneity, and hence support the assessement and comparison of their performance. The approach extends measurements from existing purely geometric concepts to physical modelling to further the links between 3-D microstructural characterization and material performance.

This new measurement method consists in applying the ECF method to a 3-D discrete representation of the heterogeneous structure provided by skeleton-based partitioning to sequentially probe the pathways to each TPB site, within each phase separately or together. The capability of the accessible TPB to characterize differences among materials and to extract additional information on the factors that control material performance and reliability compared to that of standard metric and topological properties is illustrated on composite SOFC/SOEC electrode materials and artificial packed sphere structures with controlled properties. The coherent sensitivity to local geometry and topology is tested using the differences in microstructures in the dataset and further, by local and targeted manipulation of the material. 


\section{Methodology}

\subsection{The concept of accessible TPB}

The access to TPBs in a heterogeneous material depends upon the transport phenomena and the morphology and topology of the complex 3-D pathways within the phases. A simplified 2-D schematic of this problem in Figure 1a illustrates the concept. The measure of the number or length of TPBs, based on 2-D micrograph or 3-D reconstruction is known as the total TPB. In Figure 1a, the red square, blue circle and green diamonds indicate a selection of TPBs for illustration. Only part of this selection is available for electrochemical reaction, which depends upon several conditions. The two TPBs indicated by the red square and blue circle belong to the subset of connected TPBs, i.e., they have connected pathways to the exterior of the material within all the phases. However, the TPB connected through the red pathway has lower accessibility than the blue one, because of narrower constrictions and higher tortuosity. In real heterogeneous materials, the pathways to the TPBs are not single as in the 2-D simplification shown in Figure 1a, but they are multiple, three dimensional, and reticulate.

\section{Figure 1}

Section 2.4 and 2.5 provides the detailed description of the accessible TPB measurements within the phases and how the combined and total accessible TPB are obtained. The accessible TPB within the phases is the measure of the effective conductivity $\sigma$ of the ionic, electronic and pore transport path networks between each TPB and the electrolyte, current collection and gas channels, respectively. The upper bound is the material phase bulk conductivity $\sigma_{o}$ and the subset of connected TPBs corresponds to $\sigma>0$. The measurement of the access to each TPB yields a distribution with as independent variable the common logarithm of the conductivity ratio $\sigma / \sigma_{0}$. To prepare for the discussion of results, Figure $1 \mathrm{~b}$ is intended to provide an intuitive view of the information conveyed by accessible TPB 
measurements, using selected limiting cases. A value of $\log _{10}\left(\sigma / \sigma_{\mathrm{o}}\right)$ of zero corresponds to the limiting case of an idealized material made of a superposition of materials, where each phase will conceptually occupy all the volume. Further, all TPBs are equally accessible, which yields a distribution with a single infinite peak at zero ( $\mathrm{i}$ in Figure 1b). In the case of a material made of an assembly of dense blocks and with the simplification of one-dimensional transport, the peaks are shifted towards smaller values of $\log _{10}\left(\sigma / \sigma_{\mathrm{o}}\right)$ (ii in Figure $1 \mathrm{~b}$ ). Note that the distinction between the directions in which the TPBs are connected is neglected for presentation purpose. In a real heterogeneous material designed for increased TPB length, the distribution is expected to be shifted further to the left and have a non-zero variance, because the morphology and topology of each phase is not ideal for transport (iii in Figure 1b). Therefore, distributions characterized by a mean value closer to zero and by a low standard deviation are intuitively indicative of a microstructure that provides high accessibility to TPB sites and that is uniformly utilized. It is therefore beneficial to maximize performance and reduce vulnerability against degradation phenomena that alter the morphology and topology of the material phases and the spatial distribution of the TPBs, such as coarsening and reduction-oxidation cycling of $\mathrm{Ni}$ in $\mathrm{Ni}-\mathrm{YSZ}[6,8,16-18]$.

The concept of accessible TPB is therefore not restricted to geometrical considerations. For instance, electronic conductivity is typically higher than ionic conductivity in SOFC/SOEC electrode materials, and low accessibility can cause gas-phase transport limitations. The accessible TPB is proposed to provide superior information on the factors that control material performance, over that provided by standard measurements, such as total and connected TPB, phase size distributions, coordination numbers, tortuosity or contiguity, among others. The TPB tortuosity and the TPB critical pathway radius that were introduced recently by Jorgensen et al. to quantify the "quality" of TPB sites are purely geometric concepts [24]. They convey partial information on TPB accessibility, i.e. they provide for 
each TPB, the shortest path and the smallest constriction that must be passed through [24]. In contrast, the accessible TPB integrates the differences between the transport losses along the specific pathways in each phase and is fully informed on the reticulate nature of the pathways. It therefore instrinsically accounts for the effects of the TPB tortuosity and the TPB critical pathway radius on 3-D transport.

\subsection{Imaging and standard characterization of 3-D real and artificial heterogeneous structures}

The dataset used for the development of the accessible TPB measurement comprises three types of 3-D heterogeneous structures (see Table 1). The two types of analyzed real structures are SOFC/SOEC hydrogen and oxygen electrode materials. The 3-D data consists of a Niyttria-stabilized zirconia (YSZ) sample imaged by XNT and a lanthanum strontium manganite-yttria-stabilized zirconia (LSM-YSZ) sample imaged by FIB-SEM serial sectioning. The sample preparation, imaging parameters, data reconstruction and segmentation are described in Appendix A. Six Ni-YSZ volume samples with a size of $4.675^{3}$ $\mu \mathrm{m}^{3}$ (8.5 nm voxel size) and two LSM-YSZ volume samples with a size of $5^{3} \mu \mathrm{m}^{3}(10 \mathrm{~nm}$ voxel size) have been extracted from the segmented data and their accessible TPB computed. The total conductivity of a sample from the same batch at $800^{\circ} \mathrm{C}$ in dry air $(5 \%$ relative humidity (RH) at $\left.25{ }^{\circ} \mathrm{C}[31]\right)$ was measured by the 4-point method for comparison with ECF predictions (Appendix A).

\section{Table 1}

3-D structures can be generated numerically with direct control over several metric and topological properties. The in-house Matlab code described in Ref. [28] was used to generate volumes made of randomly dispersed spherical particles that comprise three phases; the void and the two solid phases. The volume samples were created with varying volume fractions and neck sizes to verify that the developed accessible TPB measurement method has 
consistent and sufficient sensitivity to such variations in the microstructure. Monosized starting particles with a radius of 15 voxels were first deposited with the constraint of contact with at least one other particle. Then, the averaged neck size of the first solid phase was optionally adjusted by dilation. Cubic volume samples with 420 voxels on a side were created, which corresponds to the representative volume element size reported in the literature for the connected TPB in packed sphere structures [32]. For presentation purposes, the voxel size was set arbitrarily to yield a sample volume of $6^{3} \mu \mathrm{m}^{3}$.

Volume fractions, contiguity, and connected triple-phase boundary length were computed with Matlab versions of the methods developed in ref. $[4,14,15]$. Phase size distribution was measured based on the phase volume that can be filled with overlapping spheres of a given size, as described in e.g. [6,33]. The procedure implemented in Matlab consisted in the thresholding of the phase distance map followed by dilation, sequentially repeated for an inversely sorted list of grayscale values of the distance map, i.e., decreasing test sphere radii. The neck size distribution was obtained simultaneously by applying the labelling procedure used for standard contiguity measurement at each thresholding/dilation iteration. The number and neck size of the connections was obtained by counting and tracking how separate regions connect throughout the sequential process.

\subsection{Electrochemical fin model}

The ECF modelling approach has been developed as a rapid screening tool with sensitivity to local microstructure and topology [26-29]. Its application to the characterization of $\mathrm{SOFC} / \mathrm{SOEC}$ materials requires a representation of their structure as interconnected networks of segments. Upon appropriate simplifications, the segments can be approximated as axisymmetric fins with profiles for which an analytical solution to the combined charge transport and linearized surface charge transfer exists. Figure 2 and this section provide an 
overview of the method in the view of measuring accessible TPB. The analytical solutions for fins with variable cross-sectional area and the effects on material performance can be found in refs. [26-28]. Application to the computation of the effective conductivity and polarization resistance of artificial and real structures with comparison with experiments and finiteelement simulations is available in refs. [18,28,29].

\section{Figure 2}

The discrete representation of the electrode structure is obtained by image processing. An automatized tool implemented in Matlab, with calls to Avizo skeletonization, watershed transform and meshing functions, automatically executes the steps shown in Figure 2. The input is the 3-D segmented data (Figure 2a). Skeleton-based partitioning is first applied to each material phase separately (Figure $2 b$ ). The 3-D skeleton is computed from thinning of the phase's distance map. The edges (or branches) of the skeleton are used as markers for a watershed-based partitioning of the structure, which yields a representation of the structure as a network of segments. A complementary partitioning using the skeleton vertices (or nodes) as markers is performed to identify the locations of the necks in the segments. The combination of the obtained information is illustrated in the two lowest inserts of Figure $2 \mathrm{~b}$. A segment identified by edge-partitioning is highlighted in green and translucency on the left. Vertex-based partitioning of the same region yields two particles (red and blue in translucency on the right) and their interface corresponds to a neck in the structure. All the segments identified by edge-based partitioning are then sub-partitioned at their necks (results shown in Figure 2c). For convenience, these sub-segments are simply referred to as segment in the following description.

The inputs for the ECF simulations are the length, inter- and intra-phase interfacial surface areas and the total TPB length that are measured for each individual segment. In the sense of the ECF simplification, the end surfaces are flat and their area smaller than that of the 
real intra-phase interfacial surfaces. Comparison with finite-element simulations has shown that the area of the projection of the inter-phase interfacial surface on the plane normal to the skeleton at the end node is a better estimate of a segment's end surface $[28,29]$. The characterization of each individual segment serves to identify the idealized axisymmetric fin shape that best approximates its transport properties and surface electrochemistry, among those shown in Figure 2c. At present, analytical solutions have been derived for spherical, conical and hyperbolic segments [26-28]. In this study, shape fitting follows the nondimensional approach described in Ref. [29]. It stems from casting the one-dimensional governing equation (see Appendix B) in terms of the dimensionless potential $\varphi^{*}$ and two dimensionless areas $A_{c}^{*}$ and $A_{s}^{*}$ :

$$
\frac{d^{2} \varphi^{*}}{d z^{* 2}}+\frac{1}{A_{c}^{*}} \frac{d A_{c}^{*}}{d z^{*}} \frac{d \varphi^{*}}{d z^{*}}-\frac{L}{\sigma_{o} R A_{c}^{*}} \frac{d A_{s}^{*}}{d z^{*}} \varphi^{*}=0
$$

Where $\sigma_{o}$ is the bulk material conductivity $\left(\mathrm{S} \mathrm{m}^{-1}\right), R$ is the area specific charge transfer resistance $\left(\Omega \mathrm{m}^{2}\right)$ and $L$ the length of the segment $(\mathrm{m})$. The coefficients of the second and third left-hand side terms are two dimensionless parameters. The first contains information on the variation of the cross-sectional area along the segment length and is referred to as the sintering quality. The second is a resistivity ratio that informs on the balance between the resistance to charge transport and transfer. The assumption of constant sintering quality and/or resistivity ratio greatly simplifies the derivation of analytical solutions (see Appendix B). For the measurement of the accessible TPB as defined in Section 2.4, the sink/source term expressing electrochemical surface reaction equals zero. In this limiting case, the enforcement of a constant sintering quality yields segments with exponentially-varying radius profiles. With this approach, the effective conductivity of the segment is calculated from its measured length, end surface areas and bulk material conductivity. The resolution of problems with 
fixed dirichlet boundary conditions imposed at faces and/or at the location to be probed within the volume sample such as shown in Figure $2 \mathrm{~d}$ then reduces to that of a resistive network.

Comparisons between ECF and finite-element predictions of the effective conductivity of artificial packed sphere and real structures have shown that the error caused by enforcing a constant sintering quality is practically below $30 \%$, while adequate sensitivity is retained [28]. Therefore, the results presented in this manuscript should be considered as a systematic close to worst-case scenario, which does not preclude the formulation and analysis of the proposed accessible TPB measurement. Higher accuracy can be achieved by improved shape fitting, but the formulation of the accessible TPB is not affected.

\subsection{Computation of accessible TPB}

The accessible TPB is measured using the capability of the ECF approach to probe heterogeneous structures, because of the low computational requirement. A set of ECF problems is solved for each TPB in the structure, which is illustrated in Figure 3. Analysis of the accessible TPB in a cubic volume sample therefore requires the computation of the resistance to transport between the TPB and target planes within three networks, which can be performed in six directions. Figure $3 \mathrm{a}$ indicates the six directions and shows the case of a TPB with connection in direction five, with the target planes corresponding to the electrolyte and current collection/gas channel sides respectively on the lower and upper face of the volume sample.

\section{Figure 3}

A TPB is located on the surface of three segments, one for each phase. In the present study, the accessible TPB within one phase is measured with a spatial resolution given by the size of the segments, because in the current version of the code, dirichlet boundary conditions can be imposed only on the skeleton vertices (nodes). Therefore, within one phase, all the 
TPBs on the surface of a same segment have the same accessibility, but information on TPB accessibility is retrieved in most cases with a higher spatial resolution. Indeed, most likely only a subset of these TPBs are on a same segment within the other phases. Their combined accessibility in the three phases consequently differs, as illustrated in Figure $3 \mathrm{~b}$. The TPBs indicated by a dot and a square (as shown in the upper-left sub-figure in Figure $3 \mathrm{~b}$ ) are on the same segment within Phase I and II and have therefore the same accessibility within these phases. But they are on different segments within Phase III. Only TPBs that are on the same triplet of segments have an identical combined accessibility in the three phases.

The three ECF problems that need to be solved to determine the access of one TPB in one direction are illustrated in Figure 3c. The conductivity $\sigma$ of the network for transport between the TPB point and the target plane is computed by imposing values of the dimensionless potential $\varphi^{*}$ of 0 and 1 , respectively, at the corresponding skeleton vertices (node). The conductivity is calculated based on the distance $\mathrm{a}_{\mathrm{TPB}}$, because for each phase the ideal limiting case corresponds to the dense volume with that height (highlighted by the black box in Figure $3 \mathrm{c}$, right sub-figure). The highest conductivity among those computed for the two vertices of the segment is kept (node 1 and node 2 in Figure $3 \mathrm{~b}$ ). The process is performed for the six directions and repeated for all the TPB points in the structure (e.g. red dots in Figure 3b). TPBs located on the surface of segments in contact with a target plane require special treatment, which is schematized in Figure $3 \mathrm{~b}$ and described in Appendix C.

\subsection{Accessible TPB metrics}

\subsubsection{Accessible TPB within the phases}

The accessible TPB distribution within the phase $a T P B_{p h_{n}}(\sigma)$ is defined such that:

$$
T P B_{\text {tot }}=\int_{0}^{\sigma_{o}} a T P B_{p h_{n}}(\sigma) d \sigma
$$


Where $T P B_{\text {tot }}$ is the total TPB length (e.g. $\mu \mathrm{m}^{-2}$ ) in the structure and the independent variable $\sigma$ is the effective conductivity of the transport path between the TPBs and the target plane simulated by ECF within the phase being investigated. The upper bound is the material phase bulk conductivity $\sigma_{o}$ and the subset of connected TPBs corresponds to $\sigma>0$. The dimension of the distribution function $a T P B_{p h_{n}}$ is therefore e.g. $\mathrm{S}^{-1} \mu \mathrm{m}^{-1}$. For the direct comparison of the effects of local geometry and network topology on transport to TPBs in 3D heterogenous structures with varying bulk material properties, a normalized probability density function $a T P B_{p h_{n}}^{*}$ is defined:

$$
1=\int_{-\infty}^{0} a T P B_{p h_{n}}^{*}(X) d X
$$

The independent variable $\mathrm{X}$ of the accessible TPB distribution is the common logarithm of the conductivity ratio $\sigma / \sigma_{o}$ within the phase. The density function $a T P B_{p h_{n}}^{*}(X)$ therefore provides the fraction of the total TPB that has accessibility within the range $[X, X+d X]$, within the investigated phase. The following description and presentation of the results is based exclusively upon the normalized $a T P B_{p h_{n}}^{*}$ and is simply referred to as the accessible TPB for convenience. The density is practically constructed by binning the data from ECF transport simulations performed for each TPB, for a given phase and direction. The number of TPBs of unit voxel length $n_{n}\left(X_{i}\right)$ within a bin of accessibility $X_{i}$ with uniform width $\Delta X$ is counted.

$$
a T P B_{p h_{n}}^{*}\left(X_{i}\right)=\frac{C_{n} n_{n}\left(X_{i}\right)}{\Delta X}
$$

$C_{n}$ is a normalized constant, which corresponds here to the inverse of the total number of TPB sites of unit voxel length in the structure. 


\subsubsection{Combined accessible TPB}

The combined accessible TPB $\left(A T P B_{c_{n}}\right)$ gives an indication on the effect of transport losses through multiple material phases to a specific TPB site. It is obtained by merging of the accessible TPB within the phases $a T P B_{p h_{n}}$. The construction is similar to that of Eq. (4):

$$
\operatorname{aTPB}_{c_{n}}^{*}\left(X_{i}, X_{j}, X_{k}\right)=\frac{C_{n} n_{n}\left(X_{i}, X_{j}, X_{k}\right)}{\Delta X}
$$

Where $n_{n}\left(X_{i}, X_{j}, X_{k}\right)$ is the number of TPBs of unit voxel length within a bin of accessibility $X_{i}, X_{j}$ and $X_{k}$ with uniform and same width $\Delta X$, within phase $\mathrm{i}, \mathrm{j}$ and $\mathrm{k}$, respectively. The combined accessible TPB is best displayed as a 3-D volume with non-uniform density, where the three dimensions are the common logarithm of $\sigma / \sigma_{o}$ in each phase. Information on the combinations of TPB accessibilities most present in the structure, on the potential correlations between phases and on range ordering effects can be obtained by characterization in terms of centroid and radial distribution function.

\subsubsection{Total accessible TPB}

$a T P B_{p h_{n}}$ and $a T P B_{c_{n}}$ are relevant measurements to understand how the morphology and topology of the phases in heterogeneous materials can be manipulated individually, respectively together, to maximize the density of accessible active sites. In practical applications, however, the transport properties of the bulk material phases often vary by several orders of magnitude. In SOFC/SOEC materials, electronic conductivity is typically one to three orders of magnitude higher than ionic conductivity. Gas-phase transport is not expected to dominate at the scale of the active layer and in the range of operating conditions of SOFC electrodes, but the supply of reactants to TPB sites located in e.g. cavities accessible only through very narrow neck can be prevented because of diffusion limitation. These aspects are included in the proposed total accessible TPB measurement $\left(a T P B_{t o t_{n}}\right)$. 
The density function of the total accessible TPB $a T P B_{\text {tot }_{n}}^{*}\left(X_{i}^{\text {tot }}\right)$ is constructed similarly to the accessible TPB within the phase (Eq. 4), with the difference that the independent variable $X_{i}^{\text {tot }}$ accounts for the transport within the three phases. The calculation of $\sigma_{i}^{\text {tot }} / \sigma_{o}^{\text {tot }}$ follows the approach in simplified SOFC cell electrochemical models, with ohmic and nonohmic losses placed in series. Each TPB is considered as the single active site in the heterogeneous structure. The resistances $R_{I}, R_{I I}$ and $R_{I I I}$ shown in Figure $3 a$ are computed. They correspond to the resistance to transport of the pathways between the TPB point and the target planes for gas species, ions and electrons. $\mathrm{R}_{\mathrm{II}}$ and $\mathrm{R}_{\mathrm{III}}$ are directly provided by the ECF simulations described in Sections 2.3 and 2.4. A bulk ionic conductivity of $0.04 \mathrm{~S} \mathrm{~cm}^{-1}$ and electronics conductivity of $100 \mathrm{~S} \mathrm{~cm}^{-1}$ is considered in the calculations and are representative of YSZ and LSM at $800^{\circ} \mathrm{C}$ in air. The resistance for gas-phase transport $\mathrm{R}_{\mathrm{I}}$ (gas-phase) is calculated using analytical solutions valid for binary gas mixtures [34], which are recalled in Appendix D. Gas mixtures of $\mathrm{O}_{2} / \mathrm{N}_{2}$ and $\mathrm{H}_{2} / \mathrm{H}_{2} \mathrm{O}$ are considered for the case of the oxygen and hydrogen electrode, respectively. Calculations were performed for air or a $50 \% \mathrm{H}_{2} / \mathrm{H}_{2} \mathrm{O}$ mixture at $1073 \mathrm{~K}$ and an averaged current density of $1.0 \mathrm{~A} \mathrm{~cm}^{-2}$.

Indicators are proposed to compare the accessible TPB distributions in different structures. These are the mean squared error of accessible TPB with respect to the ideal case, i.e. mean and standard deviation of zero (Figure $1 \mathrm{~b}$ ). The same procedure is applicable to $a T P B_{p h_{n}}, a T P B_{c_{n}}$ and $a T P B_{t o t_{n}}$ since it enables the identification of distribution with a low bias and variance together:

$$
M S E=\frac{1}{n} \sum_{i=1}^{n}\left(X_{i}-X_{i}^{\text {ideal }}\right)^{2}
$$

Where $n$ is the number of connected TPB sites, $X_{i}$ refers to the independent variable of either the accessible TPB within the phase, total or combined accessible TPB and $X_{i}^{\text {ideal }}$ takes the value of zero (Figure $1 \mathrm{~b}$ and Section 2.5.1). 


\subsubsection{Segment current load}

The distribution of current computed by ECF simulations is post-processed to characterize the importance of each segment in the phase network. The aim and measurement approach resembles that of metrics that quantify the centrality of a node or edge in graph theory [30]. The betweenness (or load) of an edge is for instance measured using a shortestpath algorithm. It is the number of shortest paths between pairs of nodes in the network that comprise that edge. In this study, the concept is adapted to the physical context of accessible TPB. Segment current is considered, instead of the number of shortest paths. The starting and destination locations of interest are the nodes on a target plane and the TPBs, respectively, instead of all the pairs of nodes in the network. The load of a segment is measured according to Eq. 7, by summing the currents computed during each ECF simulation, for all the TPBs and for each material phase and direction. A linear variation of the potential is assumed along the direction being investigated to scale the ECF simulation results. Therefore, a correction factor $a_{T P B_{i}} / a$, is required because the imposed dimensionless potentials are always 0 at the TPB and 1 at the nodes on the target plane (see Figure 3c), regardless of the location of the TPB. Here, $a_{T P B_{i}}$ is the distance between the TPB $i$ and the target plane (Figure 3c) and $a$ is the edge length of the cubic volume sample.

$$
I_{s}=\sum_{T P B} \frac{a_{T P B_{i}}}{a} I_{s_{i}}
$$

$I_{S_{i}}$ is the current carried by segment $s$ to access the TPB site $i$. Distributions are obtained using a similar procedure to that for accessible TPB:

$$
I_{S_{n}}^{*}\left(Y_{i}\right)=\frac{C_{s_{n}} n_{s_{n}}\left(Y_{i}\right)}{\Delta Y}
$$


Where the independent variable $Y_{i}$ is the common logarithm of $I_{s} / \bar{I}_{s}$, that is the ratio of the current carried by the segment and the mean segment current load. $n_{s_{n}}\left(Y_{i}\right)$ is the number of segments within a bin of normalized current load $Y_{i}$ with width $\Delta Y$ and $C_{S_{n}}$ is the total number of segments that form the phase structure. Drawing the parallel with Section 2.5.3 and Figure $1 \mathrm{~b}$, distributions characterized by a mean close to zero and low standard deviation intuitively correspond to a microstructure that is utilized uniformly with potentially lowest vulnerability to changes in the network topology. The distributions are therefore analyzed using an indicator based on the mean squared error, similar to the accessible TPB.

\section{Results and Discussion}

\subsection{Accessible TPB in real and artificial structures}

The properties of the interconnected networks in heterogeneous materials depend upon the constituents, the fabrication and usage history. Part of the possible variations are large enough to be detected by the measurement of standard metric and topological properties. Table 1 indicates that the properties of the phases in the real and artificial structures selected for this study of accessible TPB vary significantly. Several properties of the artificial packed sphere structures have been controlled for validation. The computation of the median $\left(\mathrm{D}_{50}\right)$ and neck $\left(\mathrm{N}_{50}\right)$ sizes retrieves the input values used in the packed sphere code and the measured coordination numbers and contiguity are in line with the predictions from percolation theory, provided in parentheses in Table 1. The total and connected TPB correspond to values reported in the literature for similar materials $[8,35]$.

\section{Table 1}

The accessible TPB within the phases $a T P B_{p h_{n}}$ of selected LSM-YSZ, Ni-YSZ and packed spheres volume samples are shown in Figure 4. Each of the six curves in each plot represents the six directions in which a TPB can be connected. The subset of the TPB connected simultaneously in the six directions is depicted, but for the present structures, the 
results are almost identical if all the TPBs connected in each direction separately are displayed. For each phase and direction, the number of measurements, i.e. TPB sites, spans from 40,000 (Ni-YSZ) to 370,000 (LSM-YSZ). The number of ECF problems to be solved per phase and direction is however lower because the accessible TPB $a T P B_{p h_{n}}$ is measured with a spatial resolution that corresponds to the size of the segments (Section 2.3). It relates to the number of nodes in the phases, which ranges from 700 (Ni in Ni-YSZ) to 14,000 (pore in LSM-YSZ) for the dataset shown in Figure 4. Therefore, the low computational requirements of the ECF analytical method makes this measurement of accessible TPB tractable [28,29]. The dispatch on multiple CPUs is straightforward and a single evaluation for the largest phase networks comprising up to 17,000 segments requires less than $1 \mathrm{~GB}$ and approximately $8 \mathrm{~s}$ on a laptop computer (Dell m4800, 32 GB of RAM). Therefore, the most complex LSM-YSZ volume sample considered in this study can be processed within a few hours with access to a standard workstation or cluster (2 Sandy Bridge processors at $2.2 \mathrm{GHz}$, with 8 cores each and 32 GB of RAM on each node).

\section{Figure 4}

Figure 4 indicates that the accessible TPB varies by more than one order of magnitude in both real and artificial (packed sphere) structures. These results largely deviate from the ideal case illustrated in Figure $1 \mathrm{~b}$ and question the assumption in continuum electrode models. The accessible TPB does not differ significantly in the six directions in which a TPB can be connected, which suggests isotropy. Previous studies have shown that the type of real and artificial structures investigated here are close to isotropic in terms of transport transport properties [7,28]. Further, visual inspection indicates that the spatial distribution of the TPB sites is fairly uniform in all the volume samples (Section 3.2). The difference between the ratio $\sigma / \sigma_{\mathrm{o}}$ computed at the first and second node (Section 2.4 and Figure $3 \mathrm{~b}$ ) provides an 
indication of the error due to the accuracy defined by the size of the segments in the network. The computed error estimates for all studies considered were below $10^{-5}$.

The accessible TPB is also overall lower than the effective conductivity of the phase computed by ECF and denoted by the vertical dashed lines in Figure 4. Comparison between the ECF calculations and 4-point measurements of the total conductivity of LSM-YSZ at $800^{\circ} \mathrm{C}$ in air (range in gray in Figure 4a) shows a sufficient agreement, assuming a bulk conductivity of LSM of $100 \mathrm{~S} \mathrm{~cm}^{-1}$. In contrast, predictions of the effective conductivity of the solid and pore phases from percolation theory and the Bruggeman unsymmetrical treatment, respectively, prove inaccurate. Adequate sensitivity is not retained if the size of the particles of the two solid phases differ significantly, which is the case in the LSM-YSZ studied here.

The mean squared error of the accessible TPB data shown in Figure 4 is listed in Table 2 to support the analysis of each phase separately. We note here first that the accessible TPB in the entire material is a balance of all three phases, which are discussed later using the defined combined and total accessible TPB (Sections 2.5.2 and 2.5.3). Second, some care is needed when comparing accessible TPB measurements on different structures or directions, if the volume sample is far from cubic and/or very large, compared to the lateral extension of the pathways that predominantly contribute to provide access to a TPB. In the present study, the absence of risks due to such potential artifacts has been assessed by visual inspection of the pathways in the ECF results.

The three structures with lowest mean squared error of the accessible TPB are the pore phase in LSM-YSZ, the pore phase in the packed spheres volume, and the YSZ phase in NiYSZ, whereas LSM in LSM-YSZ provides the worst access to TPBs. These trends can be inferred from the observation of the distributions in Figure 4. In contrast, the reason for the higher mean squared error of phase II in the packed spheres volume compared to that of $\mathrm{Ni}$ in 
Ni-YSZ despite a higher volume fraction and effective conductivity, is at a first appraisal unclear. The volume fraction and ratio of the median phase and neck sizes $\mathrm{D}_{50} / \mathrm{N}_{50}$ of the phase II in artificial packed sphere structures has been varied from the nominal values listed in Table 1, within a range of $0.26-0.34$ and $1.68-2.16$, respectively. With these variations, the complete dataset available for this study comprises 42 phase structures and has been analyzed in terms of correlations between the mean squared error of $\boldsymbol{A T} \boldsymbol{P} \boldsymbol{B}_{\boldsymbol{p h}}$ and the metric and topological parameters listed in Table 1. The correlation coefficient is the highest with the average intra-phase coordination number (-0.86) and contiguity $(-0.85)$, lower for the volume fraction (-0.67) and the smallest for the ratio $\mathrm{D}_{50} / \mathrm{N}_{50}(0.21)$. Therefore, the lower accessibility within phase II compared to $\mathrm{Ni}$ originates from its lower contiguity and average intra-phase coordination number. However, the knowledge of such relationships provides partial information on the factors that control material performance and durability. Specific and localized modifications to the microstructure can impact the accessible TPB without being detectable by standard metric and topological properties, as will be discussed in details in Section 3.2.

\section{Table 2}

The combined accessible $\left(\boldsymbol{a T P} \boldsymbol{B}_{\boldsymbol{c}_{n}}\right)$ informs on how each TPB is accessible within the three phases simultaneously, without consideration of the differences in terms of physical transport processes. The purpose is to identify if the morphology and topology of each phase is such that their assembly results in couplings in terms of accessible TPB. Intuitively, a high accessibility can require that TPB sites are on specific features within each phase that are geometrically incompatible. A possible consequence is a high accessibility within a phase, but the availability for electrochemical reaction can still be compromised because of a transport limitation in another phase. Figure $5 \mathrm{a}$ and $5 \mathrm{~b}$ shows that such situation occurs in both Ni-YSZ and, to a lesser extent, in LSM-YSZ. 


\section{Figure 5}

The 3-D volume with variable density that represents combined accessible TPB in NiYSZ is artificially elongated in the direction denoted by the black arrows in Figure 5a. This suggests a possibly undesirable situation, where each phase affects separately the combined accessible TPB. In contrast, clusters of data points or deformation in the directions indicated by the black dots in Figure 5a are not observed. This suggests that the share of TPB that are difficult to access simultaneously in two or three phases is negligible. Similar trends are observed for LSM-YSZ and packed spheres (not shown), with the difference that the electron, then ion conducting phases affect most the combined accessible TPB, whereas the pore phase provides efficient transport. This result is consistent with the heavy, respectively thin tail in their $\boldsymbol{a T P} \boldsymbol{B}_{\boldsymbol{p h}}$ distributions (Figure 4a). The mean squared errors listed in Table 2 and the radial distribution functions from the centroid plotted in Figure $5 \mathrm{~b}$ indicate that the packed sphere and LSM-YSZ provide better combined accessible TPB than Ni-YSZ.

The total accessible TPB considers the differences in transport phenomena in the phases. The ionic conductivity is often the lowest in SOFC/SOEC materials. The breakdown of the contributions to the total accessible TPB provided in Figure 5c confirms that ionic conduction prevails for most TPBs. Therefore, the shapes of the total accessible TPB distributions in NiYSZ, LSM-YSZ and packed spheres shown in Figure 5e corresponds to that of the ion conducting phases in Figure 4. The ranking from the computed mean squared errors is also the same. The value is the smallest for Ni-YSZ, then similar for LSM-YSZ and the packed sphere volume (Table 2).

The results in Figure 5c show that the total accessible TPB of all the tested real and artificial structures is marginally affected by electron conduction, as long as the TPBs are connected. The contribution of gas-phase transport on the total accessible TPB depends upon the local operating conditions in a SOFC/SOEC stack. Under the simulated SOFC conditions 
of $1.0 \mathrm{~A} \mathrm{~cm}^{-2}$ and either air or $50 \% \mathrm{H}_{2} / \mathrm{H}_{2} \mathrm{O}$, the share of TPBs for which accessibility through the pore phase dominates is small. However, the access to $97-99 \%$ of this subset is blocked by diffusion limitation. The close-up view in Figure 5d shows that such TPBs are located for instance within a pore cavity that are connected only though a narrow neck. The skeleton (gray) shows part of the pathway to the TPBs (red). Its thickness corresponds to the local thickness, i.e. value of the pore phase distance map at the skeleton points. The display of the segments forming the pore phase of the region in translucent colors shows that the partitioning approach accurately captures the location of the constrictions.

\subsection{Sensitivity to network morphology and topology}

The capabilities of the developed accessible TPB measurement method to characterize the network morphology and topology are first tested on a LSM-YSZ volume sample of $3.5^{3}$ $\mu \mathrm{m}^{3}$, with a coarse LSM phase.

The accessible TPB within LSM significantly differs in the directions 5 (electrolyte at the top and the gas channel at the bottom) and 6 (inversely) (Figure 6a). The corresponding distribution of segment current load shown in Figure $6 \mathrm{~b}$ is spread over several orders of magnitude and does not exhibit clear peaks near values of $\log _{10}\left(\mathrm{I}_{\text {segment }} / \mathrm{I}_{\text {mean }}\right)$ of zero. This is intuitively indicative of transport in a structure that is not utilized isotropically (Section 2.5.1 and 2.5.4). In direction 5 , the current load density is larger above values of $\log _{10}\left(I_{\text {segment }} / I_{\text {mean }}\right)$ of one, compared to direction 6. Therefore, the importance of a few segments is further exacerbated in direction 5. Figure $6 \mathrm{c}$ and d display in 3-D the current carried by the edges. The thickness of the skeleton segments (edges) corresponds to the local thickness, for the region contiguous in direction 5 and 6 . The target faces are indicated by the dark gray nodes at the top of Figure $6 \mathrm{c}$ (direction 6) and bottom of Figure $6 \mathrm{~d}$ (direction 5). The distribution of the current load is not homogenous and several segments support principal pathways in both 
directions. In direction 5, the phase network is connected through only two regions (Figure 6d). The access to most TPBs therefore requires passing through two segments with constriction. One is shown in the blow-up view of Figure 6d. These segments carry a high current load and are central to the transport function of the LSM network in this test case. Consequently, the vulnerability to degradation phenomena that may alter one of these two segments is critical.

\section{Figure 6}

The prediction of segment current load in LSM-YSZ, Ni-YSZ and packed spheres confirms the presence of central segments in these structures as well. The distributions computed by summing the six directions are shown in Figure 7a-c and the corresponding mean squared errors are listed in Table 2. The three phase structures that provide the most uniform access to TPBs are the pore phase in LSM-YSZ and packed spheres and YSZ in LSM-YSZ. Their segment current load distributions are approximately centered to zero and have a low variance. In these networks, the maximum current carried by a segment is lower than 10 times the mean value. In comparison, the modal values for LSM in LSM-YSZ and Ni and YSZ in Ni-YSZ are shifted towards positive values, which indicate the presence of multiple central segments. The case of YSZ in Ni-YSZ suggests that an efficient accessible TPB does not necessarily imply the uniform utilization of the structure. Therefore, electrochemical performance and vulnerability are two specific objectives of relevance for the rational design of materials.

The presence of central segments can arise from the topology of the phase network and/or an uneven spatial distribution of TPBs. Figure 7 d,f,g are 3-D displays of the current distribution in the ion conducting phase networks in direction 1 (the upper face of the volume correspond to the electrolyte side). There is no evidence of anisotropy that would warrant a revision of the measurement method. Further inspection shows that central segments are 
found in all the phase structures, even in the artificial packed sphere volume. Figures $7 \mathrm{e}$ and $\mathrm{h}$ are 3-D views of the connected TPBs with color-coded accessibility. The spatial distribution of connected TPB is fairly uniform, even though the lower part of the Ni-YSZ volume sample contains less connected TPBs (not displayed in Figure 7e). Therefore, the presence of central segments in the structures cannot be be ascribed to the clustering of TPBs in specific regions, but originates from the morphology and topology of the phase networks.

Figures $7 \mathrm{e}$ and $\mathrm{h}$ do not reveal notable anisotropy in accessible TPB, especially along the considered direction. The TPBs form mainly open lines and closed circles in Ni-YSZ and packed sphere volume, respectively, because of the difference in morphology. Both ion conducting phases contain clusters of TPBs with a low accessibility (blue in in Figure 7e and h) located on geometrical features similar to those causing diffusion limitations (Section 3.1 and Figure 5d). These TPBs are fairly uniformly distributed in the volume sample and are mostly located on dead-end segments, i.e. with a coordination number of zero at one of the two nodes. All the materials analyzed in this study present this trend and therefore comprise connected TPBs that may be practically not available for electrochemical reactions.

\section{Figure 7}

The understanding of the reasons for the presence of central segments in a structure is of interest for the rational design of heterogeneous materials. In a simplified view, such segments differ from the others in terms of conductance and location in the network. Bivariate histograms indicating the relationship between the conductance (C) and current load of a segment are shown in Figure 8a, for selected phases (sub-figures a.I.-a.IV.) covering a broad range of segment current load mean squared errors. The correlation coefficient is the highest for the pore phase in packed spheres (0.71) and the YSZ phase in LSM-YSZ (0.40). Both are well interconnected networks that are uniformly used to access TPBs. The mean squared error of phase II in packed sphere is marginally higher, but the patterns differs significantly and is 
characterized by a low correlation $(0.14)$. The conductance of the segments is as expected more uniform in packed spheres but not unique [28]. In this case, the topology of the phase network dominates. The segment current load distribution in LSM deviates significantly from the ideal case (Figure 7b). The low correlation coefficient of 0.20 between segment conductance and current load suggests a mixed case, where the network topology also contributes. This is further supported by the low average inter-phase coordination number (2.81), compared to that of YSZ in LSM-YSZ (3.47) and pore in packed sphere (5.67) listed in Table 2.

\section{Figure 8}

The standard metric and topological properties listed in Table 2 have limited sensitivity to local 3-D geometrical features that can significantly impact the properties of heterogeneous materials. For instance, contiguity has been found to be insensitive to cracks within a phase [18]. The detection capability of the developed accessible TPB measurement are tested by selective removal of YSZ segments in Ni-YSZ, based upon the measurement of their current load. In a first case, the five segments that carry most of the current in all directions are deleted. In a second case, five regular segments, i.e. that have a coordination number larger than zero at the two nodes, are randomly selected among the first quarter of the segments sorted from the highest to the lowest carried current.

The effect of segment removal on the total accessible TPB and segment current load is shown in Figure 8 b.I and b.II. The accessible TPB within YSZ is mildly affected by random removal (mean squared error of 4.65 for pristine Ni-YSZ and 4.70 after removal). Selective removal results in a severe alteration (mean squared error of 4.98). The effect on the segment current load distribution follows a similar trend (mean squared error of 2.07, 2.15 and 2.21, respectively), except that the effect of random removal is here more pronounced. These alterations are challenging to detect with standard measurement. The volume fraction and 
contiguity are unchanged and the cumulative particle and neck size distributions vary by $1.5 \%$ at most. The decrease in the average intra-phase coordination number is lower than $0.8 \%$. Ascertaining that such subtle changes are significant is in practice difficult, even in degradation studies based on non-destructive imaging that allows the tracking of microstructural changes in a same sample.

\section{Conclusions}

The accessible TPB is proposed as a new measurement to provide detailed information on the role of transport in 3-D heterogeneous structures for material performance and durability. The measurement method and its relevance as a performance metric for the analysis and comparison of structures is illustrated on composite SOFC/SOEC electrode materials and artificial packed sphere structures with controlled properties.

The accessible TPB combines geometrical and physical considerations to characterize the transport pathways to sites available for electrochemical reaction. The measurement method processes data from 3-D imaging, such as x-ray nanotomography or FIB-SEM serial sectioning. It consists in applying an analytical electrochemical fin model to a 3-D discrete representation of the heterogeneous structure obtained by skeleton-based partitioning to sequentially probe the access to each TPB site within all the phases. The simulation data is processed into univariate and multivariate distributions that quantify respectively the accessible TPB within each phase separately, and the combined accessible TPB. Implementation of the physical differences between the transport phenomena in the phases yields the total accessible TPB. A metric based on the mean squared error of the defined accessible TPB is introduced to determine if a structure provides efficient and robust access to the reaction sites. 
In the investigated real and artificial structures, the accessible TPB is as expected isotropic but far from uniform. The spread in the ratio of effective pathway to bulk conductivity exceeds one order of magnitude. The analyzed structures exhibit significant differences. The correlation with standard properties is the highest for the intra-phase coordination number and contiguity, lower for the volume fraction and the smallest for the ratio $\mathrm{D}_{50} / \mathrm{N}_{50}$. However, these overall trends imperfectly characterize the relationships between microstructure and accessible TPB. Indeed, standard averaged measurements are insensitive to the removal of a few selected segments in the structure. The significant detrimental effect is in contrast clearly detected by the measurement of the accessible TPB and segment current load distribution.

The share of TPBs that have low accessibility within simultaneously two or three phases is negligible. The combined accessible TPB is therefore mostly affected by each phase separately. In the case of SOFC/SOEC electrode materials, the total accessible TPB is largely dominated by the ion conducting phase. However, diffusion limitation can passivate connected TPBs that are located for instance within a pore cavity accessible only though a narrow neck. Under the simulated conditions, the effect remains marginal since the share of such TPBs is below $3 \%$.

Central segments, i.e. those that support a significant share of the pathways to the TPB, are present in all the investigated structures, even in artificial packed sphere structures. Analysis based upon segment current load and conductance measurements highlights the distinct or combined effects of local geometry and network topology, i.e. of the conductance of the segment and the location of the segment within the network, repsectively. Selective removal of a few central segments shows the potential vulnerability of such heterogeneous materials to even minor degradations of their microstructure, which are hardly detectable by standard metric and topological properties. The improvements in detection and characterization 
capabilities provided by the developed accessible TPB measurements are of relevance for the design of heterogeneous materials.

\section{Acknowledgements}

Financial support from the National Science Foundation (Award CBET-1134052) and an Energy Frontier Research Center on Science Based Nano-Structure Design and Synthesis of Heterogeneous Functional Materials for Energy Systems (HeteroFoaM Center) funded by the US Department of Energy, Office of Science, Office of Basic Energy Sciences (Award DESC0001061) are gratefully acknowledged. Portions of this research were carried out at the Advanced Photon Source supported by the U.S. Department of Energy, Office of Science, Office of Basic Energy Sciences, under contract No. DE-AC02-06CH11357. Funding from the European Union's Seventh Framework Programme (FP7/2007-2013), project ENDURANCE (grant agreement 621207) and SOPHIA (grant agreement 621173) is acknowledged. The authors would like to thank Dr. J.A. Schuler (Hexis) for providing the LSM-YSZ samples and Thibault Fovanna for the preparation for FIB-SEM serial sectioning. Computations were perfomed using EPFL Scientific IT and Application Support Center, cluster Bellatrix.

\section{Appendix}

\section{A. Experimental}

\section{a. Synchrotron X-ray nanotomography}

A Ni-YSZ SOFC anode was imaged by synchrotron X-ray nanotomography. The material has been the subject of previous studies of microstructural changes ensuing a long-term operation in short stack conditions and redox cycling based on 2-D and 3-D imaging $[8,18]$. Anode and electrolyte bilayers were produced by tape co-casting and co-sintering resulting in a thickness of the layers in the range of $250 \mu \mathrm{m}$ and $7 \mu \mathrm{m}$, respectively. The starting 
composition of the anode layer was $55 \mathrm{wt} \%$ of nickel oxide and $45 \mathrm{wt} . \%$ of $8 \mathrm{YSZ}$ and the microstructure is the same in the supporting and electrochemically active regions.

Cylindrical samples with a diameter of approximately $10 \mu \mathrm{m}$ and a height of approximately $15 \mu \mathrm{m}$ were extracted from the anode support using a FIB lift-out technique and mounted on an invar pin [36,37]. X-ray absorption contrast measurements were performed at beamline 32-ID-C of the Advanced Photon Source (APS, Argonne National Laboratory). In the present case, imaging across the Ni K-edge white line peak (8348 eV) proved sufficient to distinguish the pore, YSZ and Ni phases [18]. At this energy, the zone plate used for the measurements provides a spatial resolution of around $20 \mathrm{~nm}$ [38]. 721 projection images with a pixel size of $8.5 \mathrm{~nm}$ were acquired during the rotation of the sample over a $180^{\circ}$ range. These were first aligned manually using the particles placed on top of the samples as fiduciary marks. The iterative algebraic reconstruction technique (i-ART) implemented in TXM wizard was then used for reconstruction [39]. The total volume for analysis is $240 \mu \mathrm{m}^{3}$, with an irregular shape.

\section{b. FIB-SEM measurements}

The second type of real structure data is a LSM-YSZ SOFC cathode (Hexis, Switzerland) imaged by FIB-SEM serial sectioning. The ink comprising LSM and YSZ powders with a ratio of 1 (vol.) was screen-printed on a 3YSZ substrate. After sintering, the thickness of the LSM-YSZ layer was in the range of $50 \mu \mathrm{m}$. A first sample was used for 4-point conductivity measurement at $800^{\circ} \mathrm{C}$ in dry air $\left(5 \%\right.$ relative humidity $(\mathrm{RH})$ at $25^{\circ} \mathrm{C}$ [31]) as a verification of ECF calculations. Another pristine sample was impregnated with resin and a volume sample from a region close to the surface was imaged by FIB-SEM serial sectioning (Zeiss NVision 40). Sufficient contrast between the LSM and YSZ phases was obtained with the energy selective backscatter (EsB) detector and an acceleration voltage of $1.6 \mathrm{kV}$, which limits the escape depth of the backscattered electron. The section pixel size and slice thickness 
were both of $10 \mathrm{~nm}$. Fiducial marks were milled before the image acquisition to adjust the position of the FIB beam and maintain the thickness variation of the slices below the nanometer. The marks consisting of groves orthogonal to the imaged plane were then used to align the sections with a Fiji script. The resulting 3-D data is a stack with a volume of of $10 \times 10 \times 15 \mu \mathrm{m}^{3}$ and an isometric voxel size of $10 \mathrm{~nm}$.

\section{c. Segmentation of the imaged data}

An anisotropic diffusion filter was first applied to the grayscale data. Segmentation of the grayscale 3-D data is required before the measurement of the accessible TPB, to obtain a digitized representation of the materials, where all the voxels that form a material phase are assigned a same label. This was performed using Matlab routines with calls to Avizo for image gradient and watershed transform computations. The LSM-YSZ data obtained by FIBSEM serial sectioning could be processed without modification of the standard workflow of watershed-based segmentation. In the case of the Ni-YSZ XNT data, the material phases could be clearly distinguished by visual inspection, but the accurate segmentation of YSZ and

pore could not be achieved by standard semi-automatic segmentation approaches. Therefore, the markers for the watershed algorithm were generated from manual segmentation. Ref. [18] describes how the consistency of the segmented Ni-YSZ data was verified based on 3-D imaging of the same sample region after reoxidation and of a pristine NiO-YSZ sample from a same batch at selected energy levels.

\section{B. Governing equation of the electrochemical fin model}

The governing equation for charge transport and surface charge transfer under the assumption of one-dimensional conduction in fins with varying cross-section is [26]:

$$
a^{2}(z) \frac{d^{2} \varphi}{d z^{2}}+2 a(z) \frac{d a}{d z} \frac{d \varphi}{d z}-\frac{2 a(z)}{\sigma_{o} R} \sqrt{1+\left(\frac{d a}{d z}\right)^{2}} \varphi=0
$$


Where $a(z)$ is the cross-sectional radius $(\mathrm{m}), \varphi$ the potential $(\mathrm{V}), \sigma_{o}$ is the bulk material conductivity $\left(\mathrm{S} \mathrm{m}^{-1}\right)$ and $R$ is the area specific charge transfer resistance $\left(\Omega \mathrm{m}^{2}\right)$. In the present study, the non-dimensional form is applied for all segments, which yields Eq. 1 in Section 2.3. The proposed accessible TPB measurement does not require the third right hand side term because there is no surface reaction. The sintering quality is the inverse of the second left hand side factor in Eq. 1 (Section 2.3):

$$
S Q=\left(\frac{L}{A_{c}} \frac{d A_{c}}{d z}\right)^{-1}
$$

The assumption of a constant sintering quality for each segment yields an exponentially varying radius profile. The measurement of SQ is thus reduced to that of the end surfaces of the segment $A_{1}$ and $A_{2}$ :

$$
S Q=\left(\ln \frac{A_{1}}{A_{2}}\right)^{-1}
$$

Under this assumption, the analytical solution for the dimensionless potential is:

$$
\varphi^{*}\left(z^{*}\right)=C_{1} \exp \left(-\frac{1}{2 S Q} z^{*}\right)+C_{2}
$$

Where $C_{1}$ and $C_{2}$ are constants determined by the boundary conditions.

\section{Access to TPB on segments touching the target plane}

Special treatment is applied to TPBs located on the surface of segments in contact with a target plane. The procedure described in Section 2.4 would result in an infinite conductivity. The accessibility within these specific segments is computed as the resistance of the segment's part delimited by the skeleton point the closest to the TPB and the node on the 
target plane (skeleton point and node 1 in Figure $3 \mathrm{~b}$ ), using the analytical solutions for the non-dimensional case. A region of interest for the measurements is complimentarily defined. The particle diameter of the coarsest phase is set as the default value for the exclusion zone. Variations of this parameter did not have a large impact on the observed trends.

\section{Diffusion overpotentials}

The calculation of diffusion overpotentials in the porous electrodes is based upon the approximation of the network of transport pathways from the target planes to the TPB as a continuum medium with effective diffusion properties. Bi-component gases are considered, i.e., $\mathrm{H}_{2} / \mathrm{H}_{2} \mathrm{O}$ and $\mathrm{O}_{2} / \mathrm{N}_{2}$ on the hydrogen and oxygen electrode sides, respectively. The effective diffusion coefficient is assumed constant, because the gas species diffusion coefficients do not differ much and the variations in partial pressure within the few microns between the target plane and the TPB remains limited for most cases. With these hypotheses and conditions, the partial pressure of the gas species at the TPB is provided by simple expressions [34]. On the hydrogen electrode side:

$$
p_{H_{2}}^{T P B}=p_{H_{2}}-\frac{R T}{2 F} \frac{a_{T P B}}{D^{e}} j
$$

Where $R$ and $F$ are the universal gas and Faraday constants, $T$ the temperature, $p_{H_{2}}^{T P B}$ and $p_{H_{2}}$ are the partial pressure at the target plane and TPB, respectively, $D^{e}$ the effective diffusion coefficient, $j$ is the current density, $a_{T P B}$ the distance between the target plane and the TPB. On the oxygen electrode side the boundary condition is not equimolar, which yields:

$$
p_{O_{2}}^{T P B}=\frac{p_{c}}{\delta_{O_{2}}}-\left(\frac{p_{c}}{\delta_{O_{2}}}-p_{O_{2}}\right) \exp \left(\frac{R T}{2 F} \frac{\delta_{O_{2}} a_{T P B}}{D^{e} p_{c}} j\right)
$$

In the present case $p_{c}$ is the atmospheric pressure, and $\delta_{O_{2}}=D_{O_{2}, k}^{e} /\left(D_{O_{2}, k}^{e}+D_{O_{2}-N_{2}}^{e}\right)$. The median pore diameter $\left(D_{50}\right)$ from phase size distribution calculation is used to estimate the Knudsen diffusion coefficient $D_{i, k}$ (Section 2.2) and the binary ordinary diffusion coefficient 
$D_{i j}$ is calculated using Fuller expression. The effective diffusion coefficients $D^{e}$ are computed by the Bosanquet relation for the anode and cathode sides:

$$
\frac{1}{D^{e}}=\frac{1}{\xi}\left(\frac{1}{D_{i, k}}+\frac{1}{D_{i j}}\right)=\frac{1}{D_{i, k}^{e}}+\frac{1}{D_{i j}^{e}}
$$

Here, $\xi$ is the computed accessible TPB within the pore phase (Section 2.5.1). The diffusion overpotentials are then for the case of an anode:

$$
\eta=-\frac{R T}{2 F} \ln \left(\frac{p_{H_{2}}^{T P B}}{p_{H_{2} O}^{T P B}}\right)
$$

And for the case of a cathode:

$$
\eta=-\frac{R T}{4 F} \ln \left(\frac{p_{O_{2}}^{T P B}}{p_{O_{2}}}\right)
$$




\section{References}

[1] M. Ebner, F. Marone, M. Stampanoni, and V. Wood, Visualization and Quantification of Electrochemical and Mechanical Degradation in Li-Ion Batteries, Science 342 (2013) 716-720.

[2] J.R. Wilson, W. Kobsiriphat, R. Mendoza, H.Y. Chen, J.M. Hiller, D.J. Miller, K. Thornton, P.W. Voorhees, S.B. Adler, and S.A. Barnett, Three-Dimensional Reconstruction of a Solid Oxide Fuel Cell Anode, Nature Materials 5 (2006) 541-544.

[3] A.P. Cocco, G.J. Nelson, W.M. Harris, A. Nakajo, T.D. Myles, A.M. Kiss, J.J. Lombardo, and W.K.S. Chiu, Three-Dimensional Microstructural Imaging Methods for Energy Materials, Phys.Chem.Chem.Phys. 15 (2013) 16377-16407.

[4] K. N. Grew, Y. S. Chu, J. Yi, A. A. Peracchio, J. R. I. Jr, Y. Hwu, F. D. Carlo, and W. K. S. Chiu, Nondestructive Nanoscale 3D Elemental Mapping and Analysis of a Solid Oxide Fuel Cell Anode, Journal of the Electrochemical Society 157 (2010) B783-B792.

[5] H. Iwai, N. Shikazono, T. Matsui, H. Teshima, M. Kishimoto, R. Kishida, D. Hayashi, K. Matsuzaki, D. Kanno, M. Saito, H. Muroyama, K. Eguchi, N. Kasagi, and H. Yoshida, Quantification of SOFC Anode Microstructure Based on Dual Beam FIB-SEM Technique, Journal of Power Sources 195 (2010) 955-961.

[6] L. Holzer, B. Iwanschitz, Th. Hocker, B. Münch, M. Prestat, D. Wiedenmann, U. Vogt, P. Holtappels, J. Sfeir, A. Mai, and Th. Graule, Microstructure Degradation of Cermet Anodes for Solid Oxide Fuel Cells: Quantification of Nickel Grain Growth in Dry and in Humid Atmospheres, Journal of Power Sources 196 (2011) 1279-1294.

[7] J. Laurencin, R. Quey, G. Delette, H. Suhonen, P. Cloetens, and P. Bleuet, Characterisation of Solid Oxide Fuel Cell Ni-8YSZ Substrate by Synchrotron X-ray Nano-tomography: from 3D Reconstruction to Microstructure Quantification, Journal of Power Sources 198 (2012) 182-189. 
[8] G.J. Nelson, K.N. Grew, J.R. Izzo Jr, J.J. Lombardo, W.M. Harris, A. Faes, A. HesslerWyser, J.Van herle, S. Wang, Y.S. Chu, A.V. Virkar, and W.K.S. Chiu, ThreeDimensional Microstructural Changes in the Ni-YSZ Solid Oxide Fuel Cell Anode During Operation, Acta Materialia 60 (2012) 3491-3500.

[9] K.L. Reifsnider, W.K.S. Chiu, K.S. Brinkman, Y. Du, A. Nakajo, F. Rabbi, Q. Liu, Multiphysics Design and Development of Heterogeneous Functional Materials for Renewable Energy Devices: the HeteroFoaM Story, Journal of the Electrochemical Society 160 (2013) F470-F481.

[10] J. Mizusaki, H. Tagawa, T. Saito, K. Kamitani, T. Yamamura, K. Hirano, S. Ehara, T. Takagi, T. Hikita, M. Ippommatsu, S. Nakagawa, and K. Hashimoto, Preparation of Nickel Pattern Electrodes on YSZ and their Electrochemical Properties in $\mathrm{H}_{2}-\mathrm{H}_{2} \mathrm{O}$ Atmosphere, Journal of the Electrochemical Society 141 (1994) 2129-2134.

[11] A. Bieberle, L.P. Meier, and L.J. Gauckler, The Electrochemistry of Ni Pattern Anodes Used as Solid Oxide Fuel Cell Model Electrodes, Journal of the Electrochemical Society 148 (2001) A646-A656.

[12] S.B. Adler, Factors Governing Oxygen Reduction in Solid Oxide Fuel Cell Cathodes, Chemical Reviews, Volume 104, Issue 10, October 2004, Pages 4791-4843

[13] P.S. Jørgensen, K. Yakal-Kremski, J. Wilson, J.R. Bowen, and S. Barnett, On the Accuracy of Triple Phase Boundary Lengths Calculated from Tomographic Image Data, Journal of Power Sources 261 (2014) 198-205.

[14] K.N. Grew, A.A. Peracchio, A.S. Joshi, J.R. Izzo Jr., and W.K.S. Chiu, Characterization and Analysis Methods for the Examination of the Heterogeneous Solid Oxide Fuel Cell Electrode Microstructure. Part 1: Volumetric Measurements of the Heterogeneous Structure, Journal of Power Sources 195 (2010) 7930-7942. 
[15] K.N. Grew, A.A. Peracchio, and W.K.S. Chiu, Characterization and Analysis Methods for the Examination of the Heterogeneous Solid Oxide Fuel Cell Electrode Microstructure: Part 2. Quantitative Measurement of the Microstructure and Contributions to Transport Losses, Journal of Power Sources 195 (2010) 7943-7958.

[16] T. Shimura, Z. Jiao, S. Hara, N. Shikazono, Quantitative Analysis of Solid Oxide Fuel Cell Anode Microstructure Change During Redox Cycles, Journal of Power Sources 267 (2014) 58-68.

[17] P.R. Shearing, R.S. Bradley, J. Gelb, F. Tariq, P.J. Withers, and N.P. Brandon, Exploring Microstructural Changes Associated with Oxidation in Ni-YSZ SOFC Electrodes using High Resolution X-ray Computed Tomography, Solid State Ionics 216 (2012) 69-72.

[18] A. Nakajo, A.M. Kiss, A.P. Cocco, W.M. Harris, M.B. DeGostin, F. Greco, G.J. Nelson, A.A. Peracchio, B.N. Cassenti, A.Deriy, S.Wang, Y.-C. Chen-Wiegart, J. Wang, J. Van herle, and W.K.S. Chiu, Characterization of Cracks and their Effects on the Effective Transport Pathways in Ni-YSZ Anodes after Reoxidation using X-Ray Nanotomography, ECS Transactions 68 (2015) 1069-1081.

[19] W.M. Harris, J.J. Lombardo, M.B. DeGostin, G.J. Nelson, H. Luebbe, J.A. Schuler, J. Van herle, J.C. Andrews, Y. Liu, P. Pianetta, Y.-C. Chen, J. Wang, and W.K.S. Chiu, Three-Dimensional Microstructural Mapping of Poisoning Phases in the Neodymium Nickelate Solid Oxide Fuel Cell Cathode, Solid State Ionics 237 (2013) 16-21.

[20] W.M. Harris, J.J. Lombardo, G.J. Nelson, B. Lai, S. Wang, J. Vila-Comamala, M. Liu, M. Liu, and W.K.S. Chiu, Three-Dimensional Microstructural Imaging of Sulfur Poisoning-Induced Degradation in a Ni-YSZ Anode of Solid Oxide Fuel Cells, Scientific Reports 4 (2014) 5246. 
[21] F. Usseglio-Viretta, J. Laurencin, G. Delette, J. Villanova, P. Cloetens, and D. Leguillon, Quantitative Microstructure Characterization of a Ni-YSZ Bi-Layer Coupled with Simulated Electrode Polarisation, Journal of Power Sources 256 (2014) 394-403.

[22] A. Bertei, B. Nucci, and C. Nicolella, Microstructural Modeling for Prediction of Transport Properties and Electrochemical Performance in SOFC Composite Electrodes, Chemical Engineering Science 101 (2013) 175-190.

[23] F. Abdeljawad, G.J. Nelson, W.K.S. Chiu, and M. Haataja, Redox Instability, Mechanical Deformation, and Heterogeneous Damage Accumulation in Solid Oxide Fuel Cell Anodes, Journal of Applied Physics 112 (2012) 036102.

[24] P.S. Jørgensen, S.L. Ebbehøj, and A. Hauch, Triple Phase Boundary Specific Pathway Analysis for Quantitative Characterization of Solid Oxide Cell Electrode Microstructure, Journal of Power Sources 279 (2015) 686-693.

[25] N. Shikazono, D. Kanno, K. Matsuzaki, H. Teshima, S. Sumino, and N. Kasagi, Numerical Assessment of SOFC Anode Polarization Based on Three-Dimensional Model Microstructure Reconstructed from FIB-SEM Images, Journal of the Electrochemical Society 157 (2010) B665-B672.

[26] G.J. Nelson, A.A. Peracchio, and W.K.S. Chiu, Analytical Investigations of Varying Cross Section Microstructures on Charge Transfer in Solid Oxide Fuel Cell Electrodes, Journal of Power Sources 196 (2011) 4695-4704.

[27] B.N. Cassenti, G.J. Nelson, M.B. DeGostin, A.A. Peracchio, and W.K.S. Chiu, Analytical Solutions for Extended Surface Electrochemical Fin Models, Journal of Power Sources 265 (2014) 282-290.

[28] M.B. Degostin, A. Nakajo, B.N. Cassenti, A.A. Peracchio, G.J. Nelson, and W.K.S. Chiu, Geometric Sensitivity of Electrochemical Fin Shape on Three Dimensional 
Microstructure Network Conductivity Analysis, Journal of Power Sources 291 (2015) 181-194.

[29] G.J. Nelson, A. Nakajo, B.N. Cassenti, M.B. DeGostin, K.R. Bagshaw, A.A. Peracchio, G. Xiao, S. Wang, F. Chen, and W.K.S. Chiu, A Rapid Analytical Assessment Tool for three Dimensional Electrode Microstructural Networks with Geometric Sensitivity, Journal of Power Sources 246 (2014) 322-334.

[30] S. Boccaletti, V. Latora, Y. Moreno, M. Chavez, and D.-U. Hwang, Complex Networks: Structure and Dynamics, Physics Reports 424 (2006) 175 - 308.

[31] J.A. Schuler, C. Gehrig, Z. Wuillemin, A.J. Schuler, J.Wochele, C.Ludwig, A. HesslerWyser, and J. Van herle, Air Side Contamination in SOFC Stack Testing, Journal of Power Sources 196 (2011) 7225-7231.

[32] C. Metcalfe, O. Kesler, T. Rivard, F. Gitzhofer, and N. Abatzoglou, Connected ThreePhase Boundary Length Evaluation in Modeled Sintered Composite Solid Oxide Fuel Cell Electrodes, Journal of The Electrochemical Society 157 (2010) B1326-B1335.

[33] B. Munch, L. Holzer, Contradicting Geometrical Concepts in Pore Size Analysis Attained with Electron Microscopy and Mercury Intrusion, Journal of the American Ceramic Society 91 (2008) 4059-4067.

[34] S.H Chan, K.A Khor, and Z.T Xia, A Complete Polarization Model of a Solid Oxide Fuel Cell and its Sensitivity to the Change of Cell Component Thickness, Journal of Power Sources 93 (2001) 130-140.

[35] G.J. Nelson, W.M. Harris, J.J. Lombardo, J.R. Izzo Jr., W.K.S. Chiu, P. Tanasini, M. Cantoni, J. Van herle, C. Comninellis, J.C. Andrews, Y. Liu, P. Pianetta, and Y.S. Chu, Comparison of SOFC Cathode Microstructure Quantified using X-ray Nanotomography and Focused Ion Beam-Scanning Electron Microscopy, Electrochem. Commun. 13 (2011) 586-589. 
[36] J.J. Lombardo, R.A. Ristau, W.M. Harris, and W.K.S. Chiu, Focused Ion Beam Preparation of Samples for X-ray Nanotomography, J. Synchrotron Rad. 19 (2012) 789796.

[37] A.M. Kiss, W.M. Harris, A. Nakajo, S. Wang, J. Vila-Comamala, A. Deriy, and W.K.S. Chiu, In Situ Heater Design for Nanoscale Synchrotron-Based Full-Field Transmission X-Ray Microscopy, Microscopy and Microanalysis 21 (2015) 290-297.

[38] J. Vila-Comamala, Y. Pan, J. Lombardo, W. M. Harris, W. K. Chiu, C. David, and Y. Wang, Zone-Doubled Fresnel Zone Plates for High-Resolution Hard X-Ray Full-Field Transmission Microscopy, J. Synchrotron Rad. 19 (2012) 705-709.

[39] Y. Liu, F. Meirer, P. A. Williams, J. Wang, J. C. Andrews, and P. Pianetta, TXMWizard: a Program for Advanced Data Collection and Evaluation in Full-Field Transmission X-ray Microscopy, J. Synchrotron Rad. 19 (2012) 281-287. 


\section{List of Tables}

Table 1. Mean metric and topological properties, median phase and neck sizes of the LSMYSZ, Ni-YSZ and packed sphere structures.

Table 2. Computed mean squared error of the accessible TPB and segment current load metrics.

\section{List of Figures}

Figure 1. (a) 2-D illustration of the access to active sites in heterogeneous electrode materials: the pathway for oxygen ions within the YSZ (gray) to the TPB indicated by the red square has higher tortuosity and a narrow neck, compared to that to the TPB point indicated by the blue circle. The TPBs indicated by the green diamond are not connected to the current collection or electrolyte side through all three phases. (b) Schematic view of accessible TPB distributions in (i) idealized heterogeneous material made of a superposition of three phase, (ii) material made of an assembly of dense blocks, with the simplification of one-dimensional transport and (iii) one phase in a real heterogeneous material.

Figure 2. Overview of the main steps for measuring the accessible TPB in real and artificial heterogeneous structures. (a) Examples of a volume samples after segmentation for each of the three types of investigated material. (b) Skeleton edge and vertex-based partitioning of the phases into segments and particles, respectively. (c) Approximation of the shape of the segments for ECF calculations. (d) Dimensionless potential $\varphi^{*}$ and current distribution obtained by ECF calculation, among the dataset created for each triple-phase boundary (points shown in red), in the three phases and for the six directions to measure the accessible TPB.

Figure 3. (a) Definition of the six directions in the accessible TPB measurements with the view of a TPB point connected in direction 5. (b) Detection of TPBs and assignment to a subsegment, schematized for phase 2 and in the lower right part, schematic of a TPB located on a segment touching a target plane. (c) Target planes and imposed boundary conditions in the three ECF simulations required to measure the accessible TPB in each phase; case of a NiYSZ volume sample and direction 5. The thickness of the skeleton edges in (c.), which corresponds to the distance map value at the skeleton coordinate points, is provided for illustration purposes.

Figure 4. Accessible TPB in (a.) LSM-YSZ, (b.) Ni-YSZ and (c.) packed sphere volume, for the six directions (1: black, 2: dashed black, 3; blue, 4: dashed blue, 5: red, 6: dashed red). The vertical dashed lines indicate the effective conductivity of the phase computed by ECF in directions 1-2 (black), 3-4 (blue) and 5-6 (red). The dashed and solid arrows corresponds to estimates of the effective conductivity provided by the Bruggeman unsymmetrical treatment and percolation theory, respectively.

Figure 5. (a) Combined accessible TPB in LSM-YSZ and Ni-YSZ. Green, blue and red isosurfaces contain respectively 90,60 and $30 \%$ of the triple-phase boundary points. The center of mass is at the intersection of the red, blue and black planes. (b) Radial distribution function computed from the 3-D data shown in (a). (c) Breakdown of the dominant contributions to the total accessible TPB. (d) Example of TPBs (red) not accessible through the pore phase (particles shown in color, skeleton shown in gray) because of a narrow neck 
causing diffusion limitation. (e) Total accessible TPB in the investigated Ni-YSZ, LSM-YSZ and packed sphere structures.

Figure 6. (a.) Accessible TPB in directions 5 and 6 (LSM in a cropped $3.5^{3} \mu \mathrm{m}^{3} \mathrm{LSM}-\mathrm{YSZ}$ volume sample) and (b.) segment current load distribution. 3-D view of segment current load in direction 6 (c.) and 5 (d.) with a close-up view of a constriction in one of the two pathways connected to face 5. The arrows in (d.) indicate which pathways in the LSM phase are connected to the target face in direction 5. The thickness of the skeleton edges in (c.) and (d.), which corresponds to the distance map value at the skeleton coordinate points, is provided for illustration purposes.

Figure 7. Segment current load distribution summed in all directions in Ni-YSZ (a), LSMYSZ (b) and packed-sphere structures (c). (d) Segment current load in YSZ in Ni-YSZ in direction 1 and (e) spatial distribution of TPBs with color coded accessibility. (f) Segment current load in YSZ in LSM-YSZ in direction 1. (g.) Segment current load in phase II of the packed sphere structure and (h) spatial distribution of TPBs with color coded accessibility.

Figure 8. Effects of network topology on material performance: (a) Relationship between segment conductance (C) and segment current load (I): phase I (a.II) and II (a.II) in packed spheres, YSZ (a.III) and LSM (a.IV) in LSM-YSZ. (b) Effect of the removal of selected skeleton segment in YSZ in Ni-YSZ on (b.I) accessible TPB and (b.II) distribution of segment current load. 


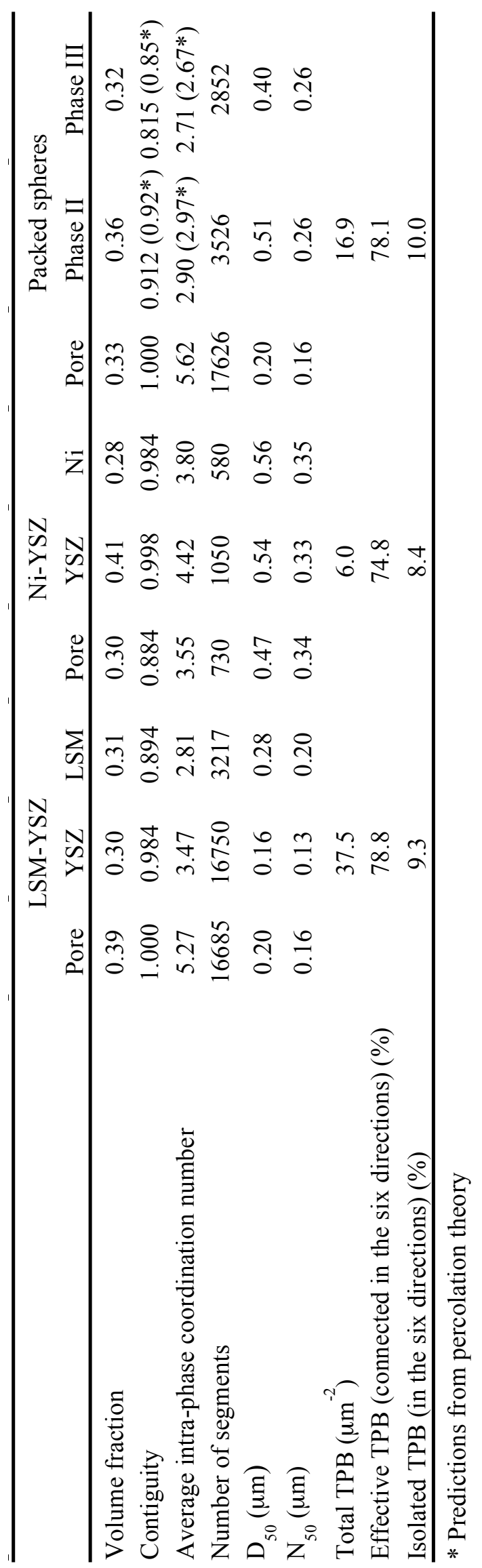




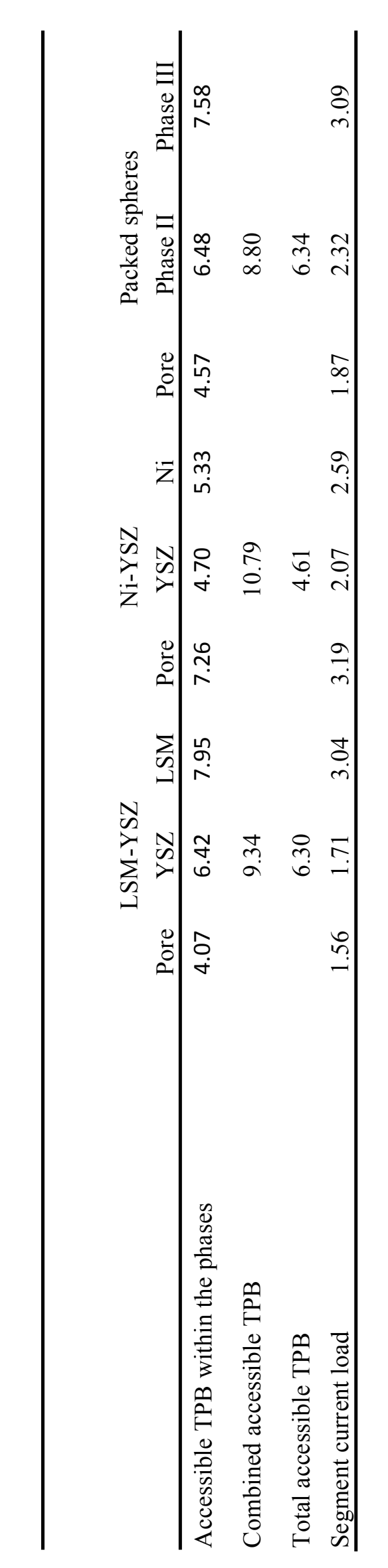



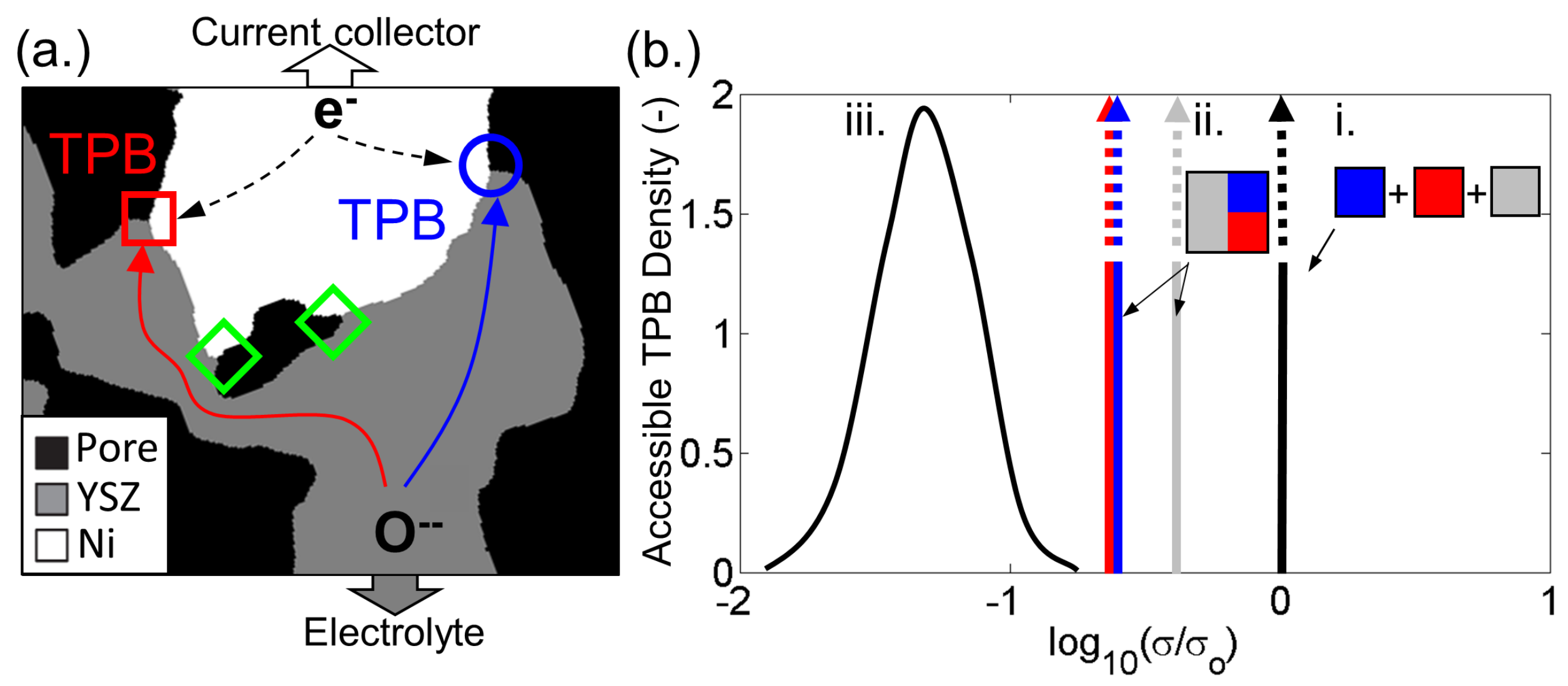


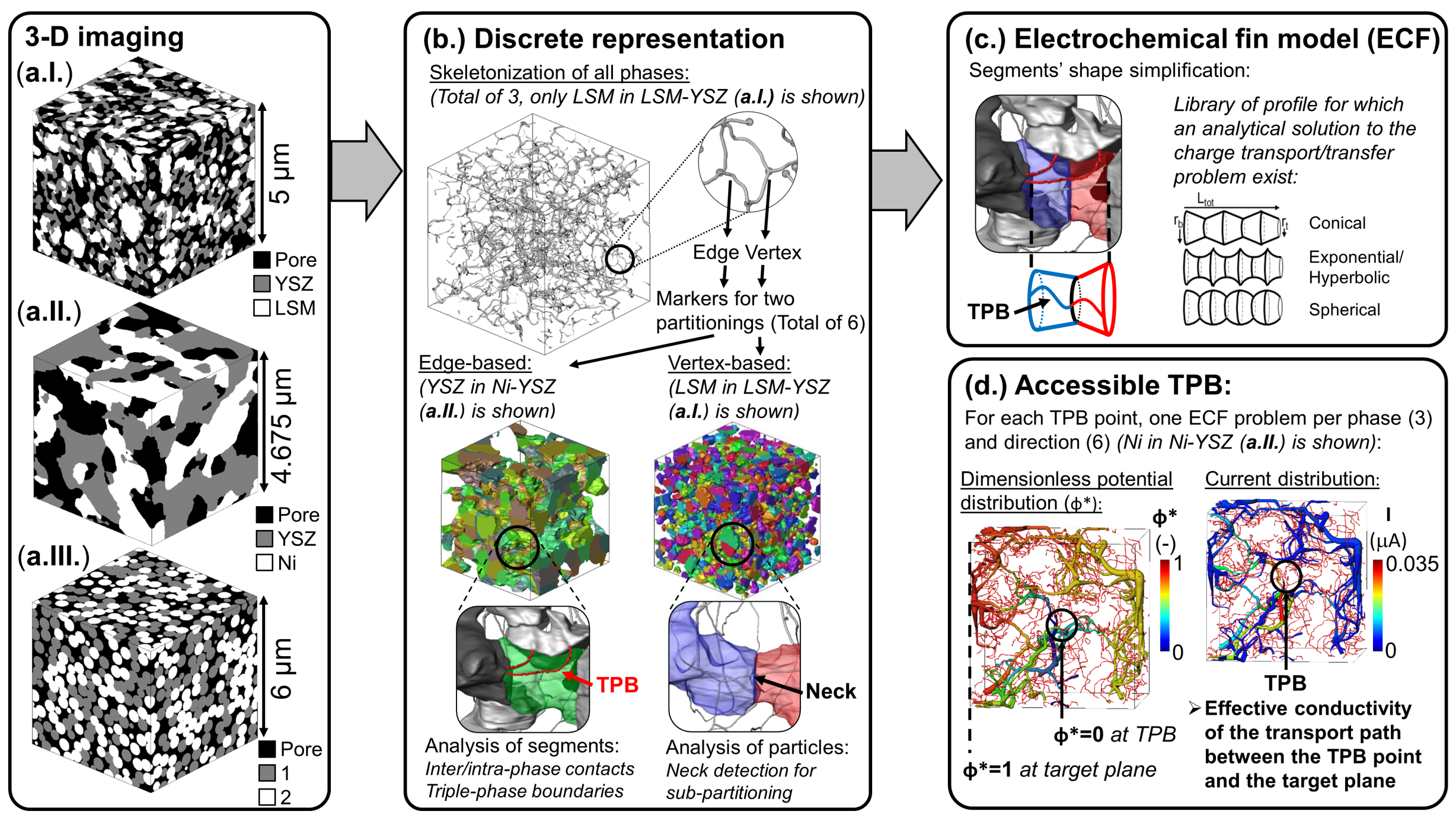



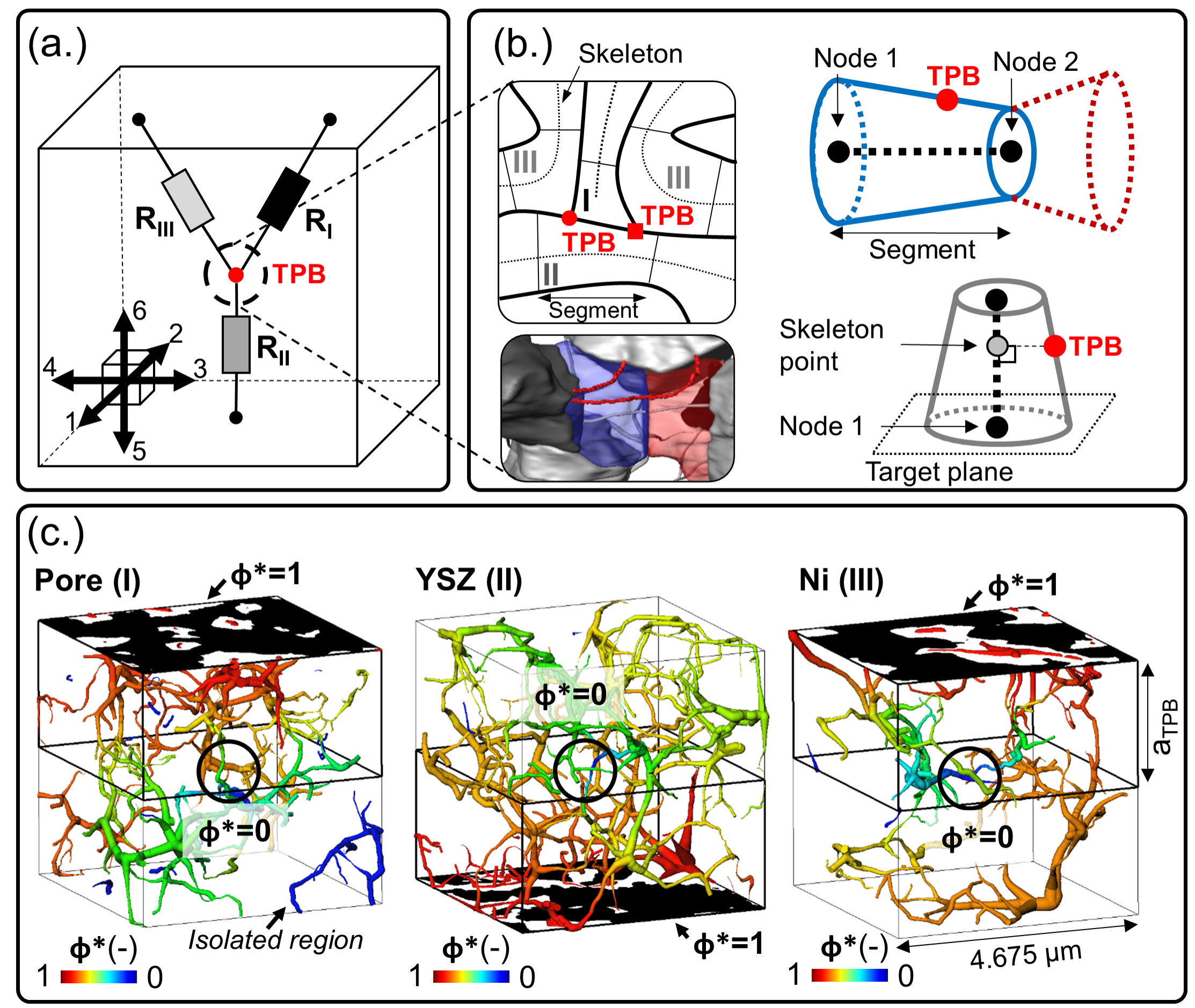

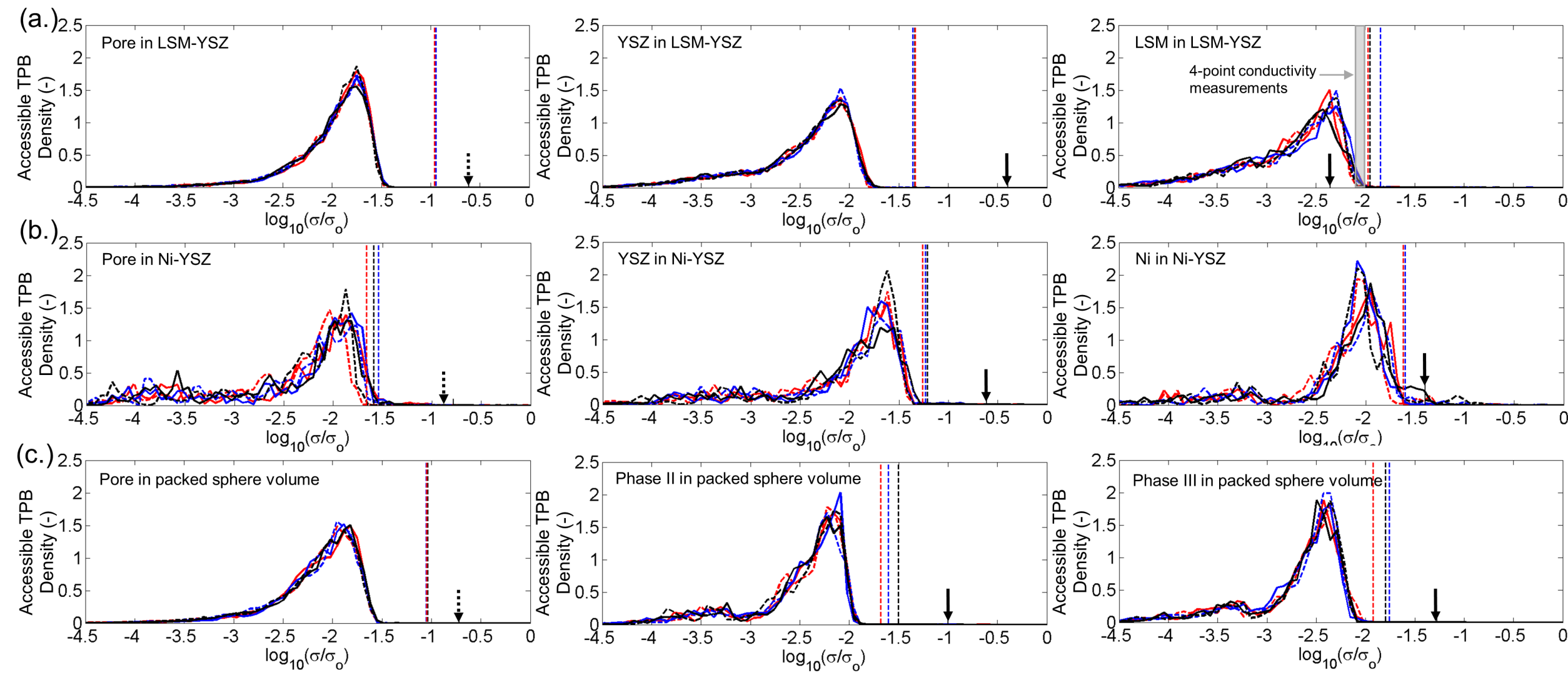

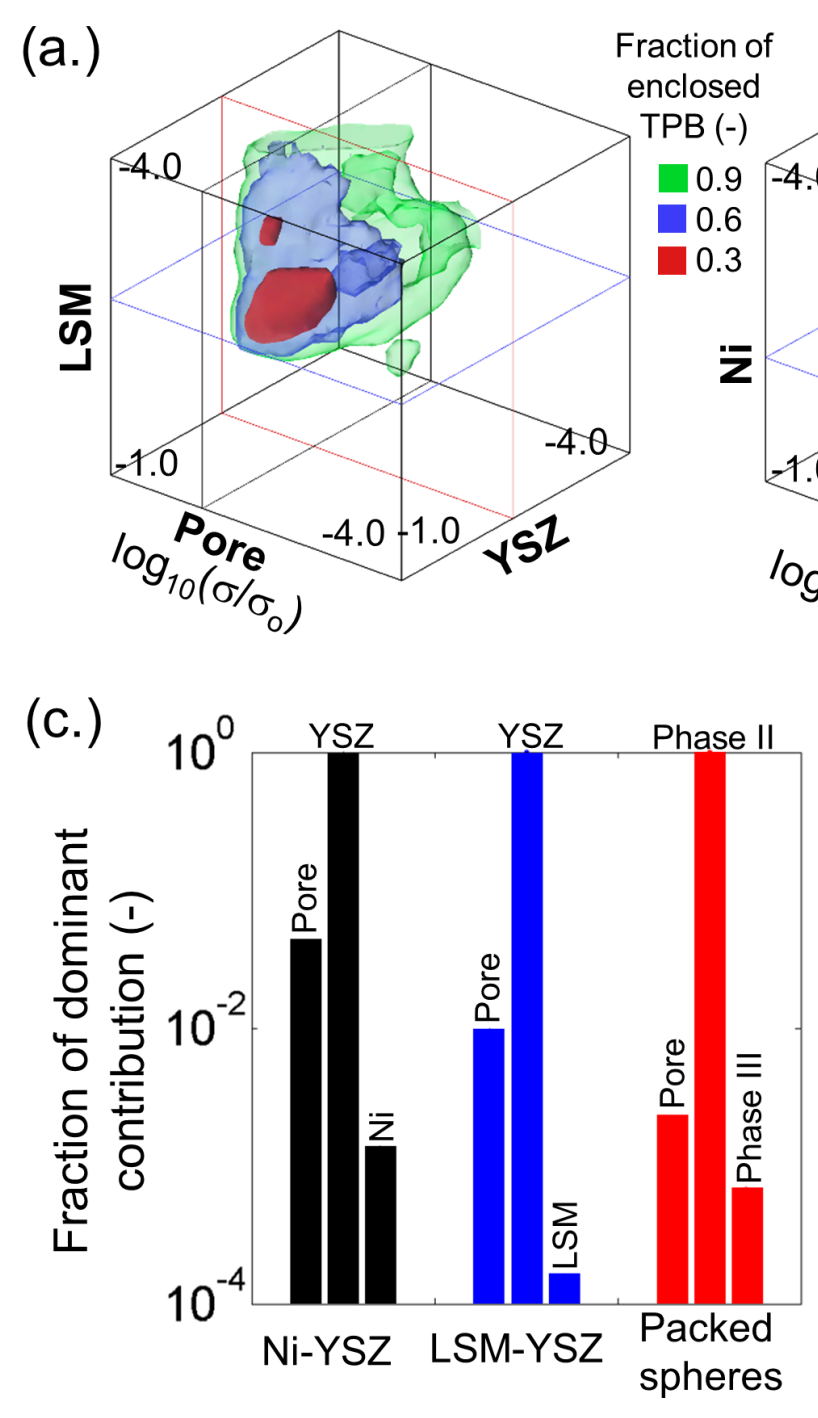

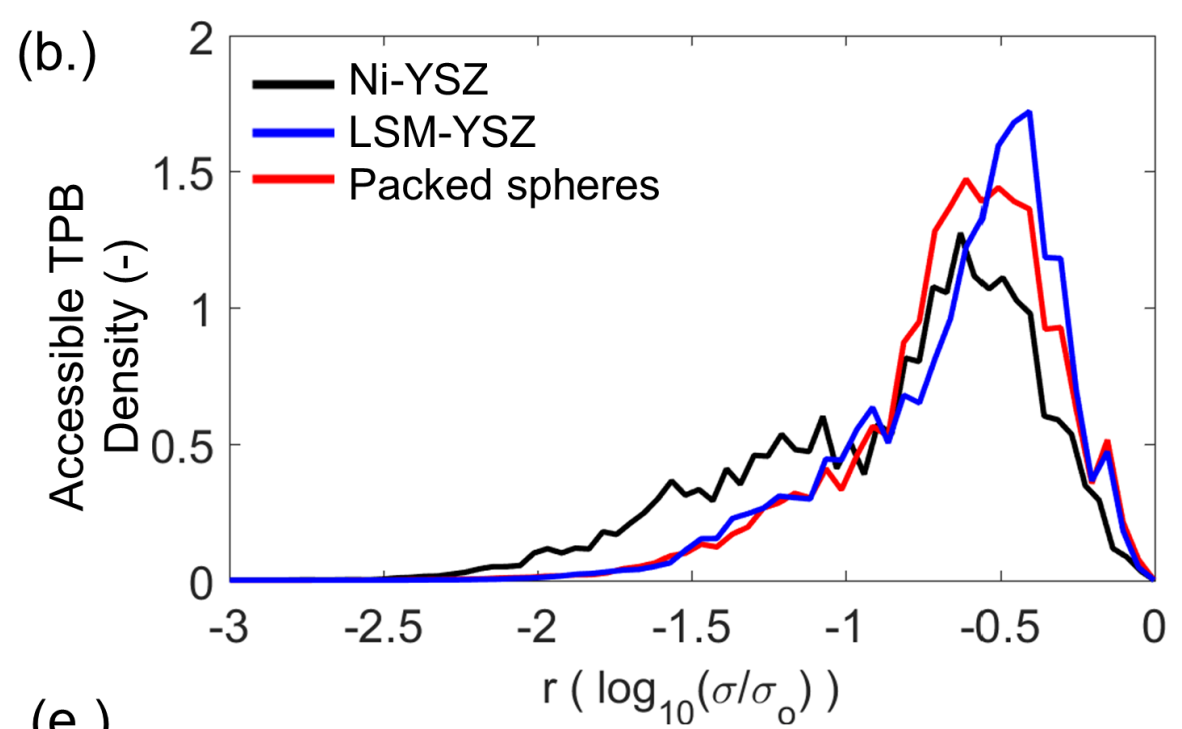

(d.)
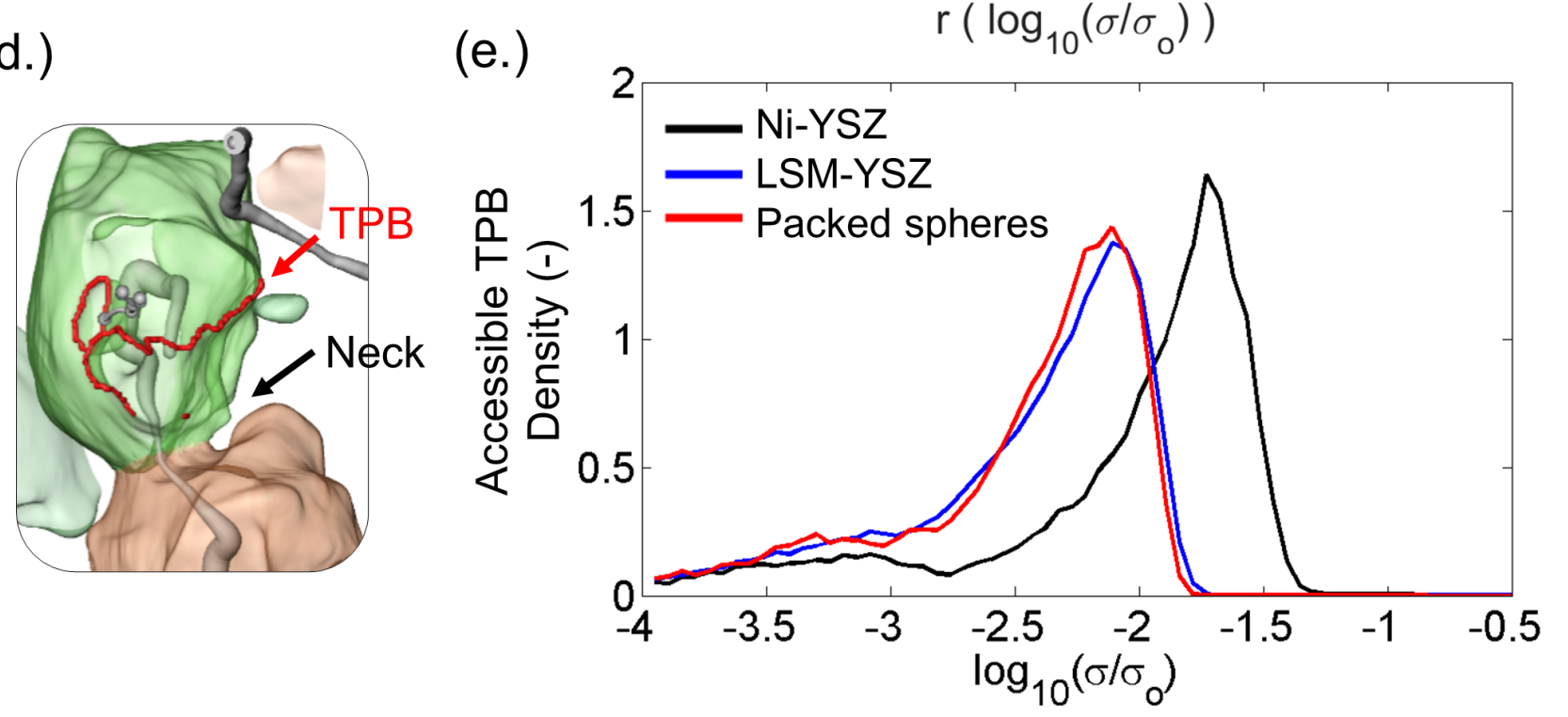


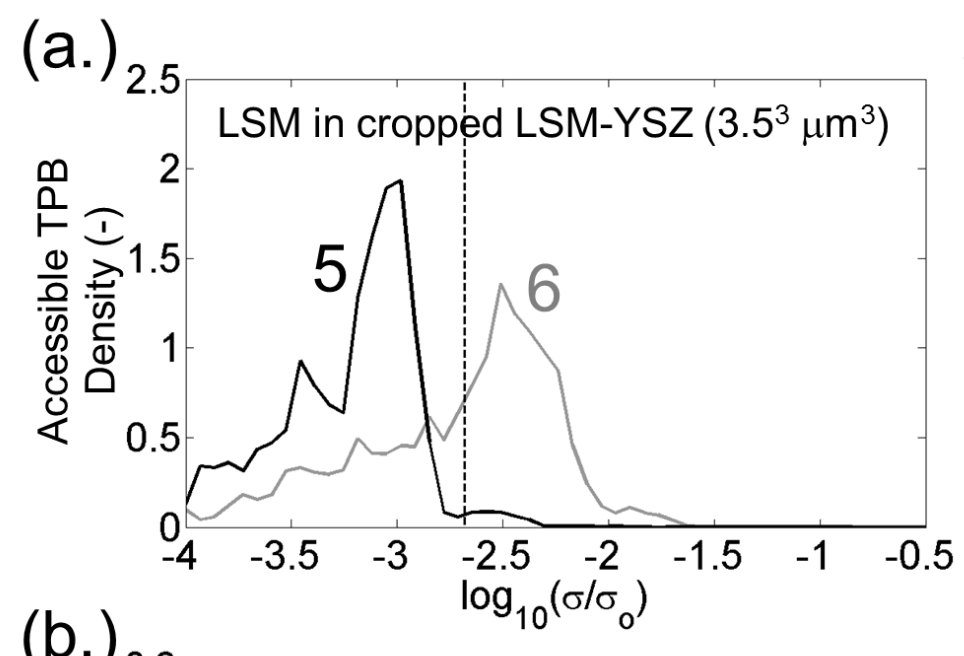

(c.) Target face

(b.) 0.6

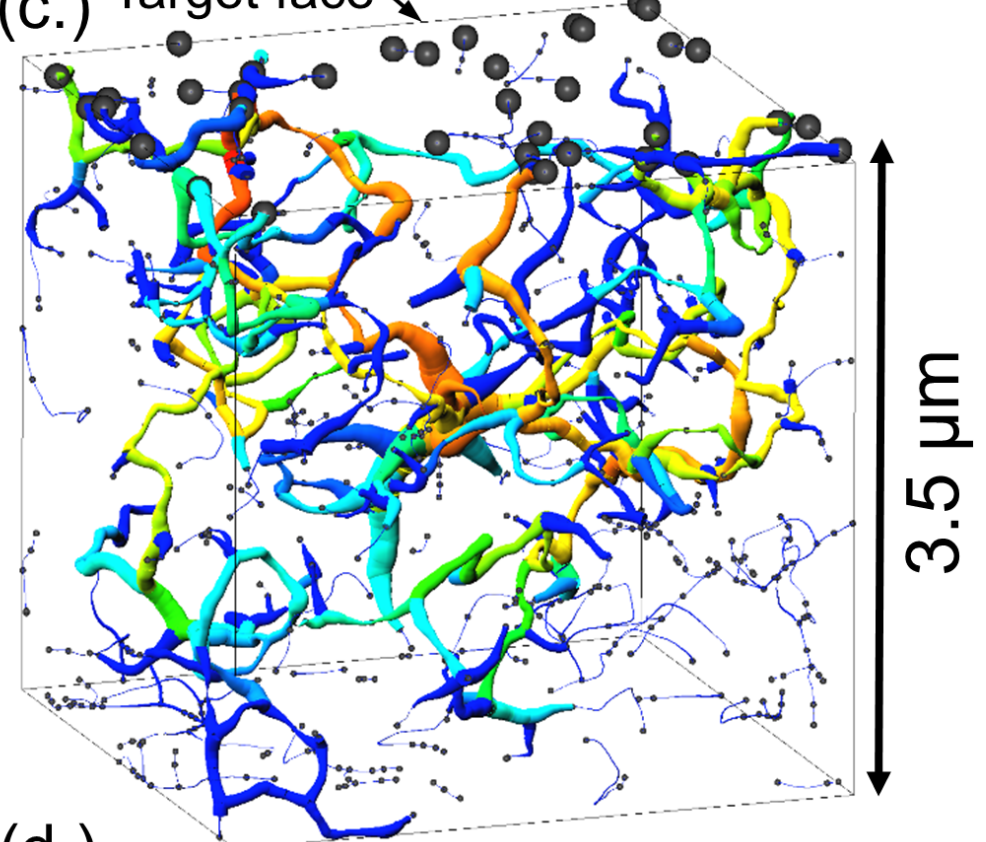

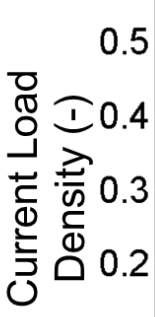

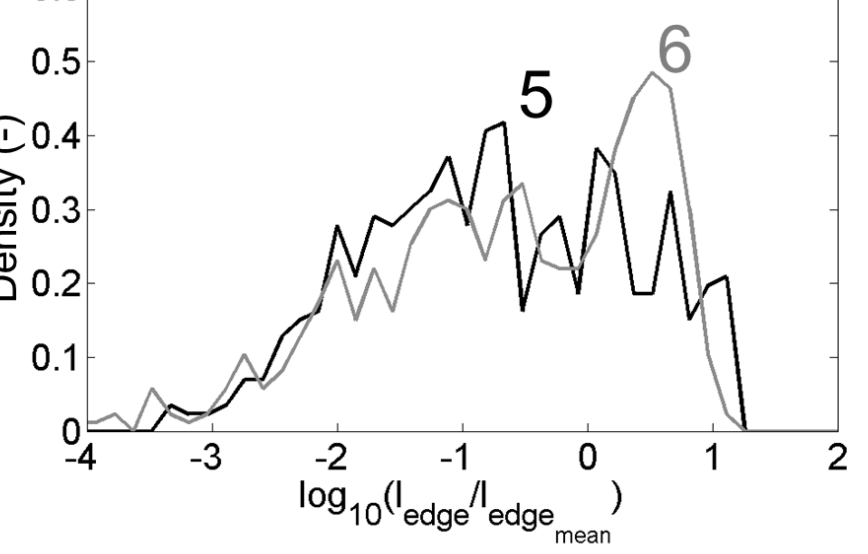

(d.)

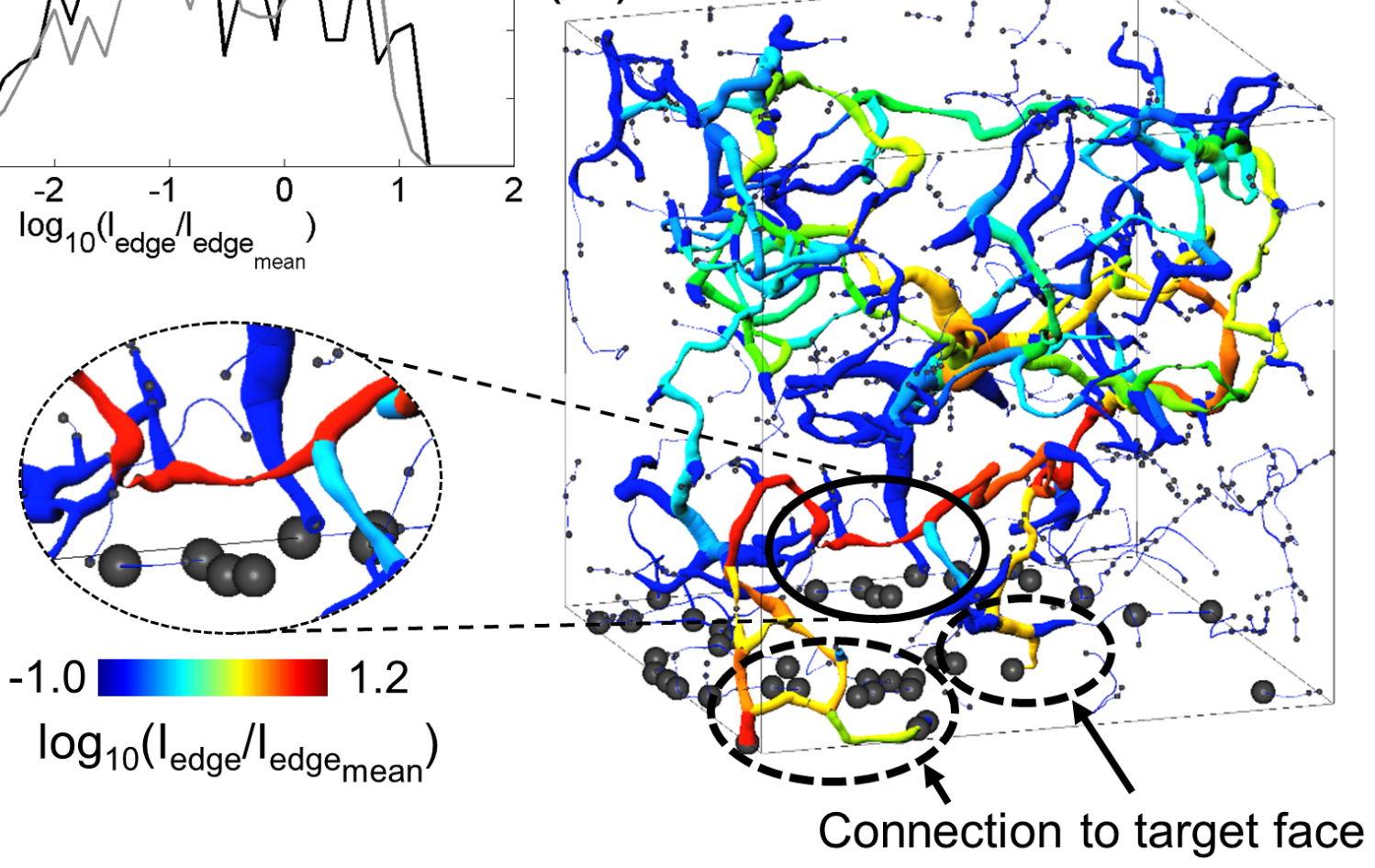



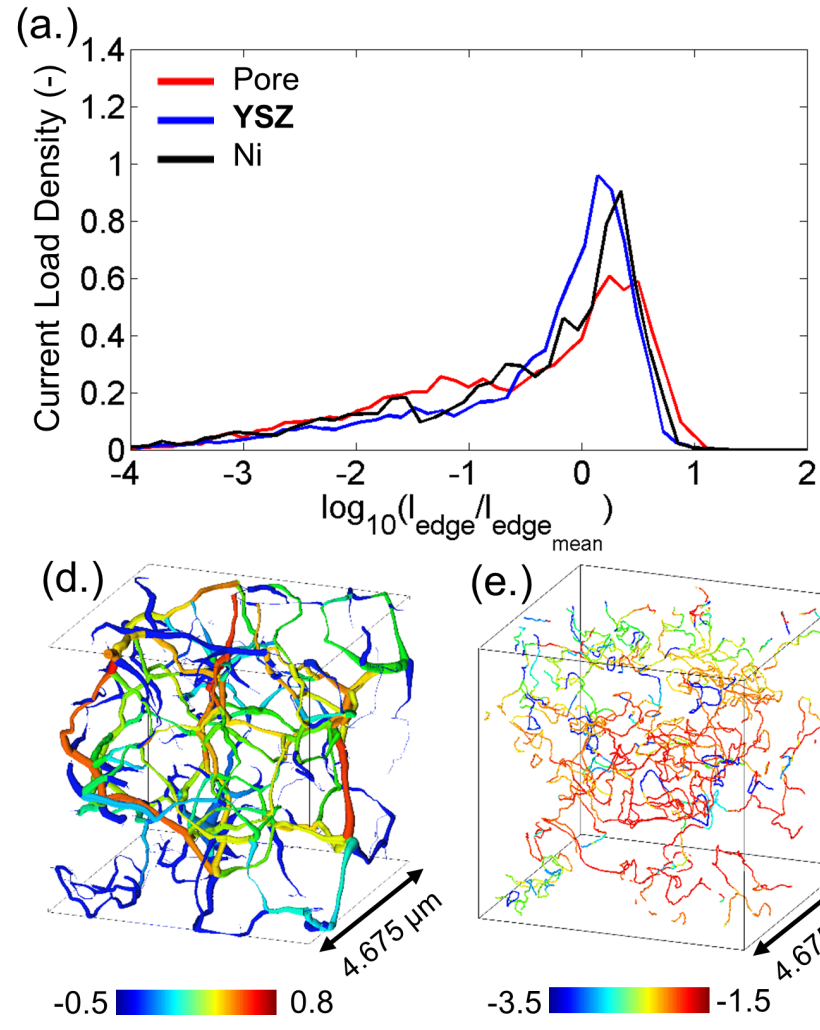

$\log _{10}\left(l_{\text {edge }} / l_{\text {edge }} e_{\text {mean }}\right)$

(e.)

$-3.5 \square-1.5$ $\log _{10}\left(\sigma / \sigma_{0}\right)$ (b.) 1.4
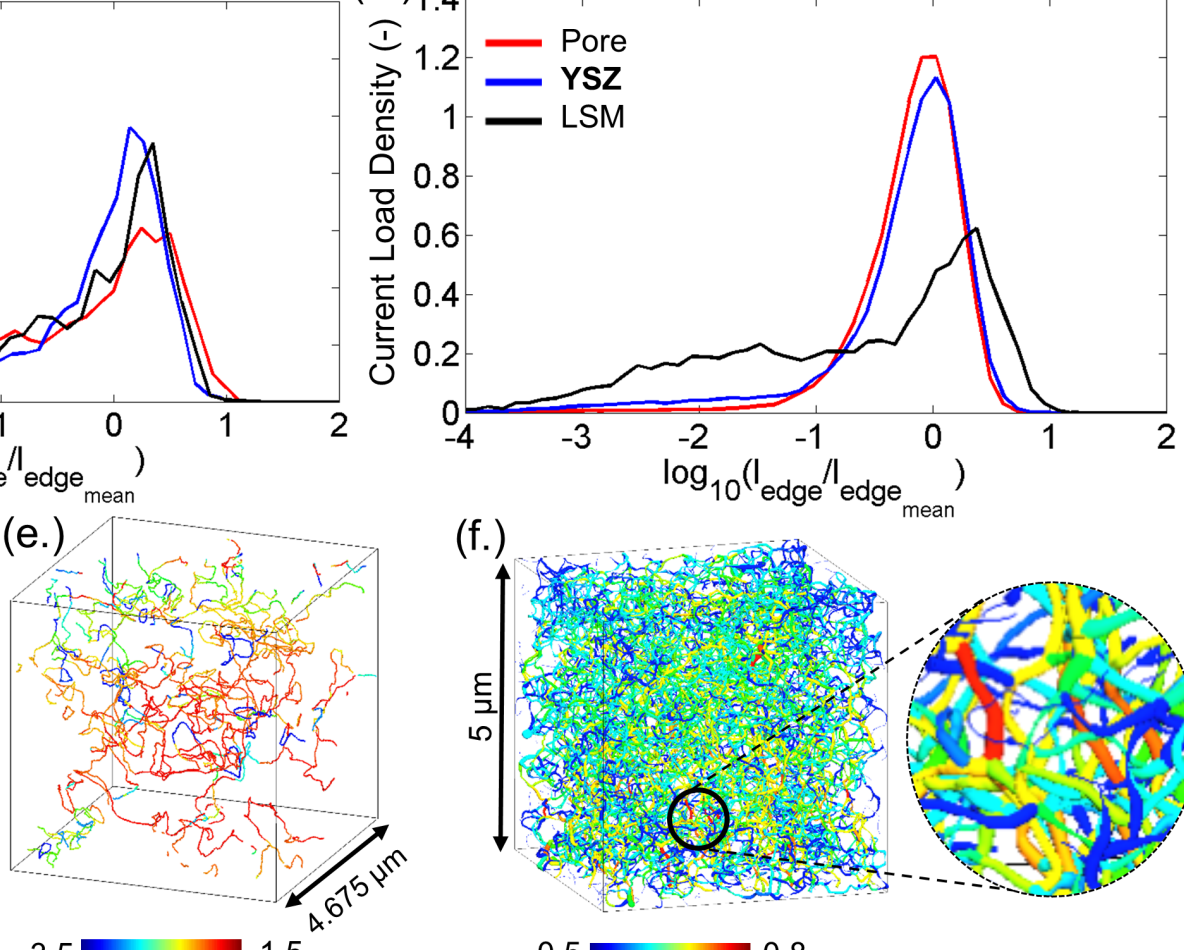

(f.)

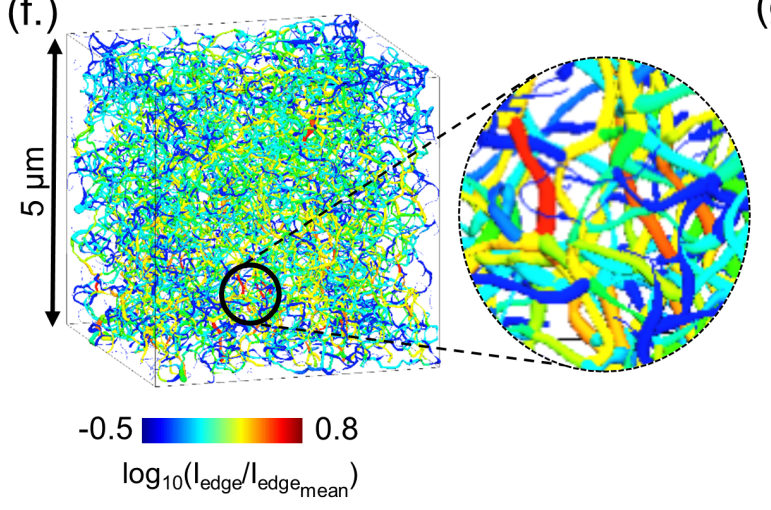

(c.) 1.4

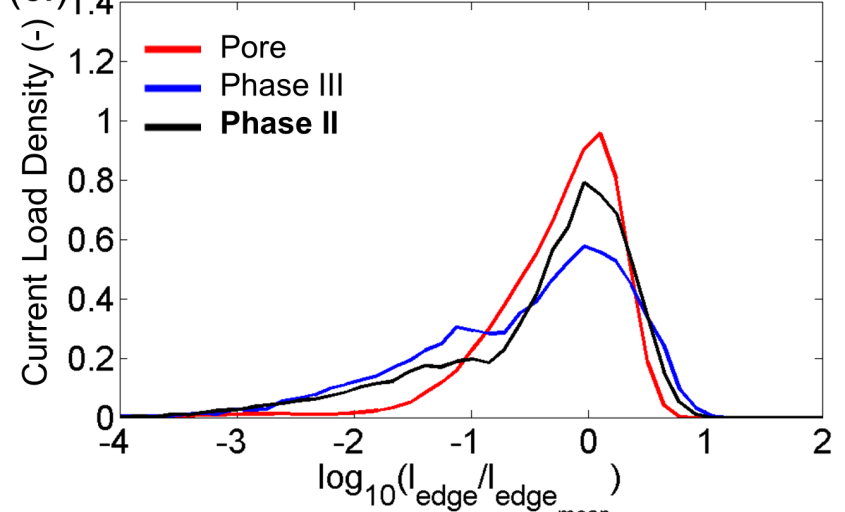

(g.)

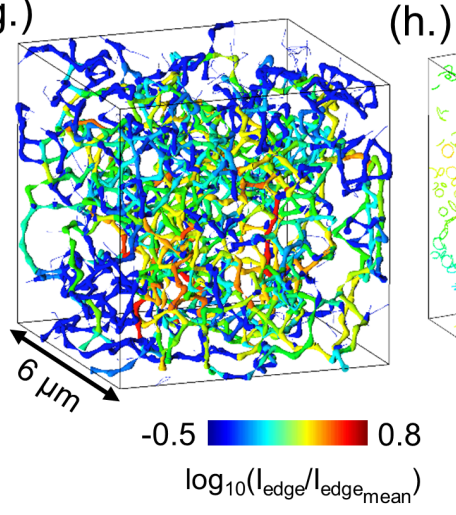

(h.)

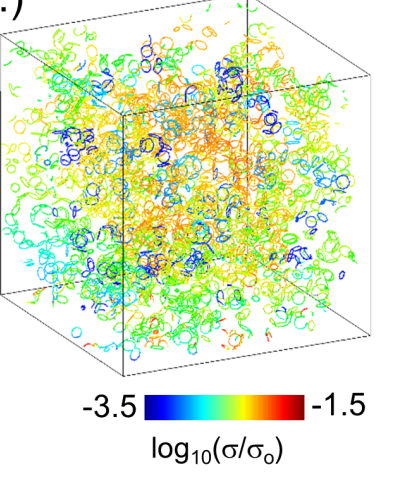



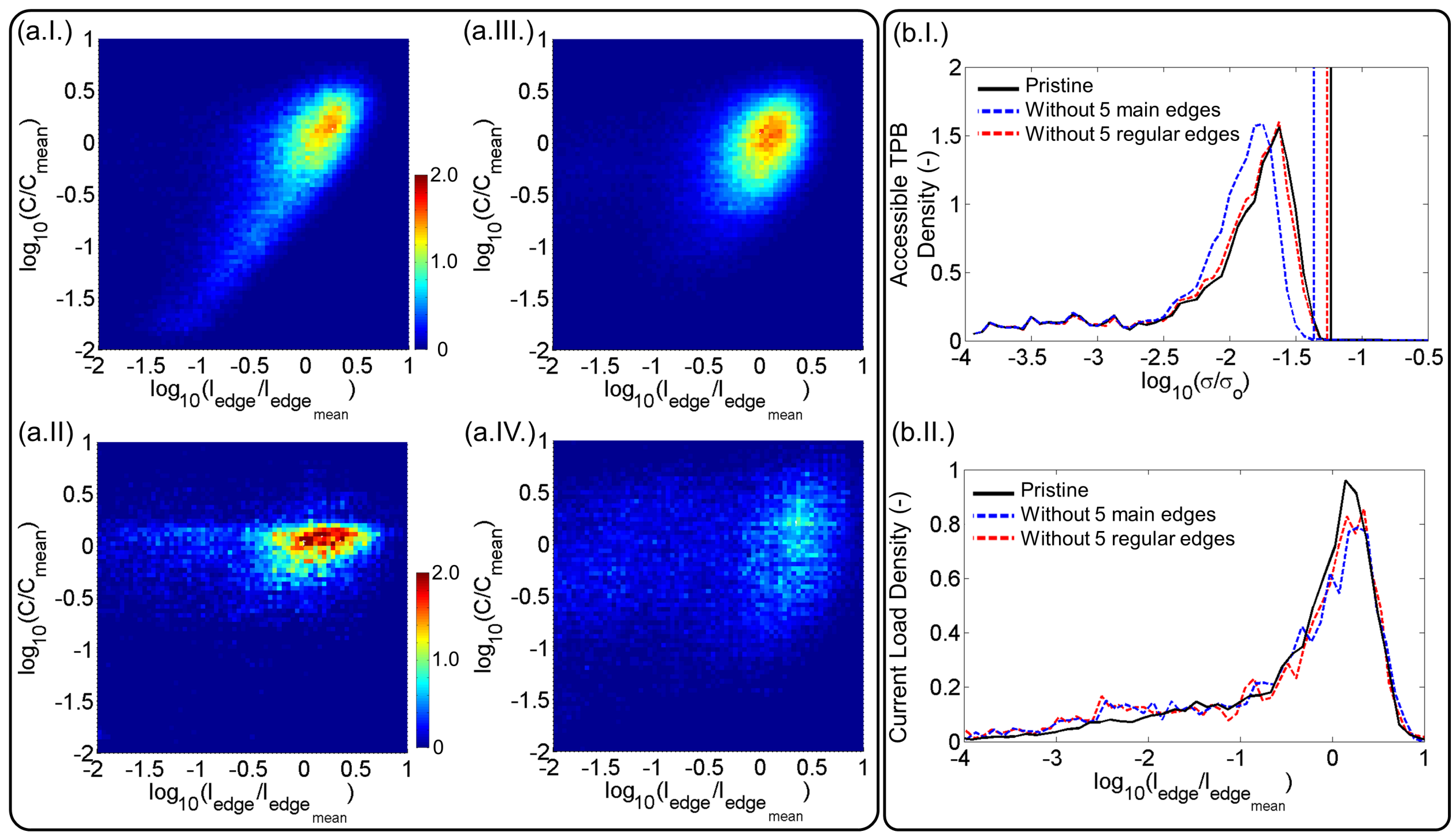

(b.II.)

I $0 .-$ Without 5 main edges

(1) W Without 5 regular edges

0.6

ॠ 0.4

0.2 